\title{
Measurements of atmospheric muon neutrino oscillations, global analysis of the data collected with MACRO detector
}

\author{
The MACRO Collaboration
}

M. Ambrosio ${ }^{12}$, R. Antolini ${ }^{7}$, D. Bakari ${ }^{2,17}$, A. Baldini ${ }^{13}$, G. C. Barbarino ${ }^{12}$, B. C. Barish ${ }^{4}$, G. Battistoni ${ }^{6, a}$ Y. Becherini ${ }^{2}$, R. Bellotti ${ }^{1}$, C. Bemporad ${ }^{13}$, P. Bernardini ${ }^{10 *}$, H. Bilokon ${ }^{6}$, C. Bloise ${ }^{6}$, C. Bower ${ }^{8}$, M. Brigida $^{1}$, F. Cafagna ${ }^{1}$, D. Campana ${ }^{12}$, M. Carboni ${ }^{6}$, S. Cecchini ${ }^{2, b}$ F. Cei ${ }^{13}$, V. Chiarella ${ }^{6}$, B. C. Choudhary ${ }^{4}$, D. Cosson $^{6}$, S. Coutu ${ }^{11, c}$ G. De Cataldo ${ }^{1}$, H. Dekhissi ${ }^{2,17}$, C. De Marzo ${ }^{1}$, I. De Mitri ${ }^{10}$, U. Denni ${ }^{6}$, J. Derkauoi ${ }^{2,17}$, M. De Vincenzi ${ }^{18}$, A. Di Credico ${ }^{7}$, C. Favuzzi ${ }^{1}$, C. Forti $^{6}$, A. Frani ${ }^{6}$, P. Fusco ${ }^{1}$, G. Giacomelli ${ }^{2}$, G. Giannini ${ }^{13, d}$

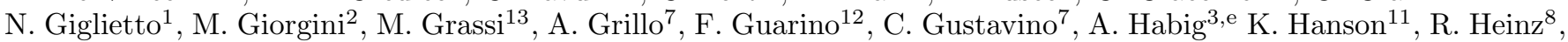
E. Iarocci ${ }^{6, \mathrm{f}}$ E. Katsavounidis ${ }^{4, \mathrm{~g}}$ I. Katsavounidis ${ }^{4, \mathrm{~h}}$ E. Kearns $^{3}$, H. Kim ${ }^{4}$, S. Kyriazopoulou ${ }^{4}$, E. Lamanna ${ }^{14, \mathrm{i}}$ C. Lane $^{5}$, D. S. Levin ${ }^{11}$, P. Lipari ${ }^{14}$, M. J. Longo ${ }^{11}$, F. Loparco $^{1}$, F. Maaroufi ${ }^{2,17}$, G. Mancarella ${ }^{10}$, G. Mandrioli ${ }^{2}$, S. Manzoor ${ }^{2, j}$ A. Margiotta ${ }^{2}$, A. Marini ${ }^{6}$, D. Martello ${ }^{10}$, A. Marzari-Chiesa ${ }^{16}$, M. N. Mazziotta ${ }^{1}$, A. Mengucci ${ }^{6}$, D. G. Michael ${ }^{4}$, S. Mikheyev ${ }^{4,7, k}$ P. Monacelli ${ }^{9}$, T. Montaruli ${ }^{1, *}$, M. Monteno ${ }^{16}$, S. Mufson $^{8}$, J. Musser $^{8}$, D. Nicolò $^{13}$, R. Nolty ${ }^{4}$, C. Orth ${ }^{3}$, G. Osteria ${ }^{12}$, O. Palamara ${ }^{7}$, V. Patera ${ }^{6}$, L. Patrizii ${ }^{2}$, R. Pazzi ${ }^{13}$, C. W. Peck ${ }^{4}$, L. Perrone $^{10}$, S. Petrera ${ }^{9}$, V. Popa ${ }^{2,1}$ A. Rainò ${ }^{1}$, J. Reynoldson ${ }^{7}$, F. Ronga ${ }^{6}$, C. Satriano ${ }^{14, m}$ K. Scholberg ${ }^{3, g}$, A. Sciubba ${ }^{6}$, M. Sioli $^{2}$, M. Sitta ${ }^{16, n}$ P. Spinelli ${ }^{1}$, M. Spinetti ${ }^{6}$, M. Spurio ${ }^{2}$, R. Steinberg ${ }^{5}$, J. L. Stone ${ }^{3}$, L. R. Sulak ${ }^{3}$, A. Surdo ${ }^{10}$, G. Tarlè ${ }^{11}$, V. Togo ${ }^{2}$, M. Vakili ${ }^{15, o}$ C. W. Walter ${ }^{3}$, R. Webb ${ }^{15}$

1 Dipartimento di Fisica dell'Università di Bari and INFN, 70126 Bari, Italy

2 Dipartimento di Fisica dell'Università di Bologna and INFN, 40126 Bologna, Italy

3 Physics Department, Boston University, Boston, MA 02215, USA

4 California Institute of Technology, Pasadena, CA 91125, USA

5 Department of Physics, Drexel University, Philadelphia, PA 19104, USA

6 Laboratori Nazionali di Frascati dell'INFN, 00044 Frascati (Roma), Italy

7 Laboratori Nazionali del Gran Sasso dell'INFN, 67010 Assergi (L'Aquila), Italy

8 Depts. of Physics and of Astronomy, Indiana University, Bloomington, IN 47405, USA

9 Dipartimento di Fisica dell'Università dell'Aquila and INFN, 67100 L'Aquila, Italy

10 Dipartimento di Fisica dell'Università di Lecce and INFN, 73100 Lecce, Italy

11 Department of Physics, University of Michigan, Ann Arbor, MI 48109, USA

12 Dipartimento di Fisica dell'Università di Napoli and INFN, 80125 Napoli, Italy

13 Dipartimento di Fisica dell'Università di Pisa and INFN, 56010 Pisa, Italy

14 Dipartimento di Fisica dell'Università di Roma "La Sapienza" and INFN, 00185 Roma, Italy

15 Physics Department, Texas A\&M University, College Station, TX 77843, USA

16 Dipartimento di Fisica Sperimentale dell'Università di Torino and INFN, 10125 Torino, Italy

17 L.P.T.P, Faculty of Sciences, University Mohamed I, P.B. 524 Oujda, Morocco

18 Dipartimento di Fisica dell'Università di Roma Tre and INFN, 00146 Roma, Italy

a Also INFN Milano, 20133 Milano, Italy

b Also IASF/CNR, Sezione di Bologna, 40129 Bologna, Italy

c Also Department of Physics, Pennsylvania State University, University Park, PA 16801, USA

d Also Università di Trieste and INFN, 34100 Trieste, Italy

e Also Physics Department, University of Minnesota, Duluth, MN 55812, USA

f Also Dipartimento di Energetica, Università di Roma "La Sapienza", 00185 Roma, Italy

g Also Department of Physics, MIT, Cambridge, MA 02139, USA

h Also Intervideo Inc., Torrance, CA 90505, USA

i Also Dipartimento di Fisica dell'Università della Calabria, 87036 Rende (Cosenza), Italy

j Also RPD, PINSTECH, P.O. Nilore, Islamabad, Pakistan

k Also Institute for Nuclear Research, Russian Academy of Science, 117312 Moscow, Russia

1 Also Institute for Space Sciences, 76900 Bucharest, Romania

m Also Università della Basilicata, 85100 Potenza, Italy

n Also Dipartimento di Scienze e Tecnologie Avanzate, Università del Piemonte Orientale, 15100 Alessandria, Italy

- Also Resonance Photonics, Markham, Ontario, Canada

* Corresponding authors: paolo.bernardini@le.infn.it, teresa.montaruli@ba.infn.it 
Received: 23 February 2004 /

Published online: 14 July 2004 - (c) Springer-Verlag / Società Italiana di Fisica 2004

\begin{abstract}
The final analysis of atmospheric neutrino events collected with the MACRO detector is presented. Three different classes of events, generated by neutrinos in different energy ranges, are studied looking at rates, angular distributions and estimated energies. The results are consistent for all the subsamples and indicate a flux deficit that depends on energy and path-length of neutrinos. The no-oscillation hypothesis is excluded at $\sim 5 \sigma$, while the hypothesis of $\nu_{\mu} \rightarrow \nu_{\tau}$ oscillation gives a satisfactory description of all data. The parameters with highest probability in a two flavor scenario are $\sin ^{2} 2 \theta_{m}=1$ and $\Delta m^{2}=0.0023 \mathrm{eV}^{2}$. This result is independent of the absolute normalization of the atmospheric neutrino fluxes. The data can also be used to put experimental constrain on this normalization.
\end{abstract}

\section{Introduction}

Bruno Pontecorvo was the first, already in 50's, to mention the possibility of neutrino oscillations, more precisely of neutrino $\leftrightarrow$ antineutrino transitions in vacuum [1]. Since then, many experimental studies of neutrino oscillations have been made with solar, reactor, accelerator and atmospheric neutrinos.

Assuming mixing of two neutrino flavors (for example, $\nu_{\mu}$ and $\left.\nu_{\tau}\right)$ and two mass eigenstates $\left(\nu_{2}\right.$ and $\left.\nu_{3}\right)$, the survival probability for muon neutrinos is

$$
P\left(\nu_{\mu} \rightarrow \nu_{\mu}\right)=1-\sin ^{2} 2 \theta_{m} \sin ^{2}\left(1.27 \Delta m^{2} L_{\nu} / E_{\nu}\right)
$$

where $\theta_{m}$ is the mixing angle, $\Delta m^{2}=m_{3}^{2}-m_{2}^{2}$ is the difference of the squares of the eigenstate masses (in $\left.\mathrm{eV}^{2}\right), L_{\nu}$ is the neutrino path-length (in $\mathrm{km}$ ) and $E_{\nu}$ is the neutrino energy (in GeV).

Atmospheric neutrinos are a unique "beam" to investigate neutrino oscillations since they cover a region of parameter space until now unexplored by man-made neutrino beams. The energies extend from fractions of $\mathrm{GeV}$ up to several tens of $\mathrm{TeV}$, and the baseline varies from about $20 \mathrm{~km}$ at the zenith to about $13000 \mathrm{~km}$ at the nadir.

An "atmospheric neutrino anomaly" was first observed during the 80's when the IMB [2] and Kamiokande [3] water Cerenkov detectors measured a $\nu_{\mu} / \nu_{e}$ ratio inconsistent with the expectation for standard massless neutrinos. The same ratio measured by calorimetric detectors (Frejus [4] and NUSEX [5]) was consistent with the expectation, but with lower statistical significance. In 1994 Kamiokande reported the zenith angle dependence of the flavor ratio for events with visible energy greater than $1 \mathrm{GeV}$ [6]. This result gave a new hint favoring neutrino oscillations.

Currently, three experiments (MACRO, SuperKamiokande, Soudan 2) indicate that the atmospheric neutrino anomaly can be explained by assuming oscillation in the $\nu_{\mu} \rightarrow \nu_{\tau}$ channel with maximal mixing and $\Delta m^{2}$ of a few $10^{-3} \mathrm{eV}^{2}$. These results were obtained using different techniques and observing events in different energy ranges. The SuperKamiokande 50 kton water Čerenkov detector presented results concerning contained, semi-contained muon and electron neutrino events [7], stopping [8] and upwardthroughgoing muons [9] with energies from about $200 \mathrm{MeV}$ up to tens of TeV. The Soudan 2 calorimetric detector, with very good electron/muon identification capability, provided further information on the deficit of muon events [10].
The study of the atmospheric neutrino oscillation was a primary goal of the MACRO project [11]. The first neutrinoinduced upward-throughgoing muons were observed between 1989 and 1994, during the detector construction, and first results were presented in 1993 [12,13] and published in 1995 [14]. The data showed a deviation compared to the predicted zenith distribution of the upgoing muon flux, with the largest deficit for vertical directions, in agreement with the hypothesis of $\nu_{\mu} \rightarrow \nu_{\tau}$ oscillation. The detector was completed in April 1994, and the presence of its upper part allowed the detection of other classes of lower energy $\nu$ events: neutrinos interacting in the absorbers in the lower part of the detector and upward-stopping muons. The analysis of upward-throughgoing muon data collected between 1994 and 1997 and the highest probability oscillation parameters $\left(\Delta m^{2}=0.0025 \mathrm{eV}^{2}\right.$ and $\left.\sin ^{2} 2 \theta_{m}=1\right)$ were published in 1998 [15], after having been presented first at the Vulcano Conference [16] and then at the Neutrino 1998 Conference [17].

Later, in 2000 lower energy results that yielded an allowed parameter region overlapping the smaller one from the upward-throughgoing muons were published [18]. In 2001 we presented results [19] that excluded matter effects such as those expected in the $\nu_{\mu} \rightarrow \nu_{\text {sterile }}$ channel at more than $99 \%$ c.l.

The oscillation hypothesis was confirmed by a recent analysis that exploited neutrino energy estimates obtained from multiple Coulomb scattering of muons [20,21].

The neutrino events collected with MACRO were also analysed to search for point-like astrophysical $\nu$-sources [22], for diffuse extragalactic $\nu$-fluxes [23] and for weakly interacting massive particles of dark matter [24].

Here the final analysis of all atmospheric neutrino topologies detected with MACRO is presented. As a conclusive paper, many details about the data sample, the analysis procedures and the statistical methods are provided. Small differences from the results reported in previous papers are due to the use of the total data sample and to a new calibration of the scintillator data.

\section{MACRO as a $\nu$ detector}

The MACRO apparatus was located in the Gran Sasso underground laboratory, with a minimum rock overburden of $3150 \mathrm{hg} / \mathrm{cm}^{2}$. It was a large rectangular box $(76.5 \times 12 \times$ $9.3 \mathrm{~m}^{3}$ ) divided longitudinally into 6 supermodules and 
vertically into a lower and an upper part, called the attico. For a full description of the apparatus see $[25,26]$. The active elements were liquid scintillation counters for time measurement and streamer tubes (ST) for tracking. The lower half of the detector was filled with trays of crushed rock absorber alternated with ST planes, while the attico was hollow and contained the electronics racks and work areas. The rock absorbers set a minimum energy threshold for vertical muons of $1 \mathrm{GeV}$.

The tracking system allowed the reconstruction of the particle trajectory in different views [26]. The intrinsic angular resolution for muons typically ranged from $0.2^{\circ}$ to $1^{\circ}$ depending on track length; the angular spread due to multiple Coulomb scattering in the rock and to kinematical angle for neutrino-induced muon was larger than this resolution. The absolute pointing capability was checked by observing the Moon's shadow in the atmospheric muon flux [27].

The scintillator system consisted of horizontal and vertical layers of counters. The total charge and the time were measured with two independent systems, ERP (Energy Response Processor) and PHRASE (Pulse Height Recorder and Synchronous Encoder), both described in [25]. The ERP data have been used for the neutrino analyses and PHRASE was used for an independent check (see Appendix C). Time and longitudinal position resolution for single muons in a counter were about $0.5 \mathrm{~ns}$ and $10 \mathrm{~cm}$, respectively. The photomultiplier signal was split into a direct output and one attenuated by a factor 10 to maintain onscale readings for very large pulses. Two different thresholds were used for the timing of these two outputs and the redundancy of the time measurement helped to eliminate spurious effects. Further details about scintillator calibrations and time measurements are in [26].

Thanks to its large area, fine tracking granularity and electronics symmetry with respect to upgoing and downgoing flight directions, the MACRO detector was a proper tool for the study of upward traveling muons. Further, it was sufficiently massive $(5.3 \mathrm{kton})$ that it also collected a statistically significant sample of neutrino events induced by internal interactions.

The streamer tubes and the scintillation counters made possible the identification of neutrino events on the basis of time-of-flight $(T o F)$ measurements, as well as by topological criteria. Four different classes of neutrino events were detected (see Fig. 1):

- UpThrough. This is the largest neutrino event sample for MACRO. It is characterized by upward-going muons crossing the detector; they originated in charged current (CC) $\nu_{\mu}$-interactions in the rock below the apparatus. The direction of flight is determined by measuring the $T o F$ given by

$$
\frac{1}{\beta}=\frac{c}{v}=\frac{c\left(t_{2}-t_{1}\right)}{l_{\mathrm{sci}}}
$$

where $t_{1}$ and $t_{2}$ are the times measured in higher and lower scintillator planes, respectively, and $l_{\text {sci }}$ is the path-length between the scintillators. Therefore $1 / \beta$ is +1 for downgoing tracks and -1 for upgoing tracks to

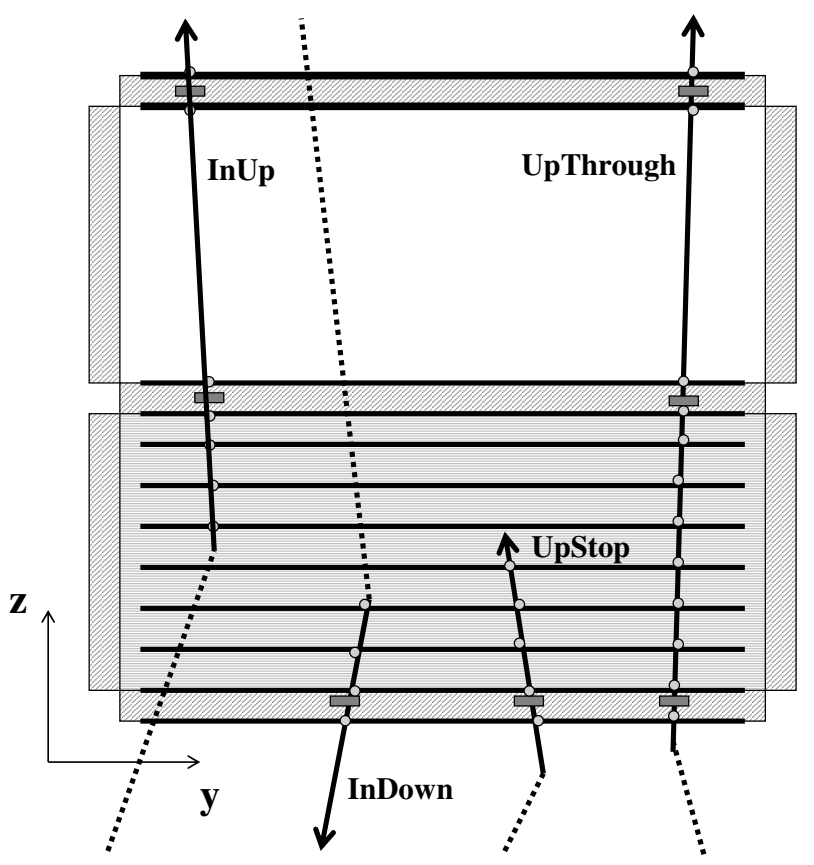

Fig. 1. Topologies of events induced by muon neutrino interactions inside or below the detector. The circles indicate the ST hits and the boxes, the scintillator hits

within measuring errors. For about $50 \%$ of the events the $T o F$ is redundantly measured by more than two scintillator layers.

- InUp. This class includes events with an upward-going track starting inside the lower part of the detector due to a $\nu$-interaction there. As for the UpThrough events, the $T o F$ is also measured for this class.

- UpStop. These events are caused by $\nu$-interactions in the rock below the apparatus producing an upgoing track which stops inside the detector. Only the lower scintillator layer is fired and so a $T o F$ measurement cannot be made.

- InDown. Events due to an internal interaction associated with a downward-going track. In this case also, only the lower layer of scintillators is crossed.

The last two classes of events are recognized by means of topological criteria. Both have a track with one end in the bottom layer of scintillator counters and the other, inside the detector. Therefore it is not possible to distinguish these topologies and they are studied together, the UpStop+InDown sample.

Figure 2 shows the energy spectra of the parent neutrinos for the three topologies, calculated with the Monte Carlo (MC) simulation described in the following (in the absence of oscillations). One can observe that the UpThrough sample is generated by a broad range of neutrinos extending from a few $\mathrm{GeV}$ to approximately $1 \mathrm{TeV}$, with a median energy of approximately $50 \mathrm{GeV}$. The other samples are generated by lower energy neutrinos that have spectra of very similar shape with median energies of approximately $2.4($ InUp) and $2.2 \mathrm{GeV}$ (UpStop+InDown) respectively. 


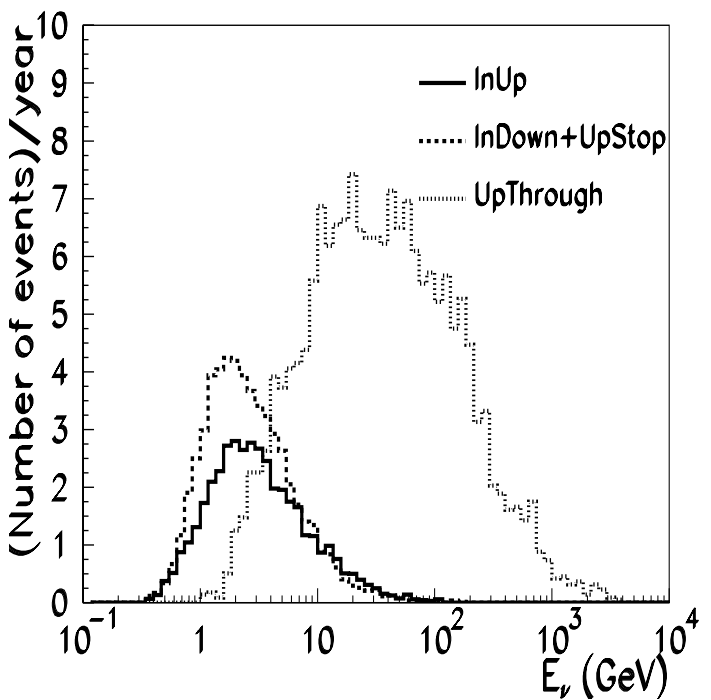

Fig. 2. Simulated distribution of the parent neutrino energy (no oscillations) giving rise to the different topologies of events. The distributions are normalized to one year of data-taking and the analysis cuts are included

\section{Data sample}

The modularity of the detector allowed data-taking with partial configurations of the apparatus, starting in March 1989. The 1-smod data were collected with the lower part of the first supermodule and the 6 -smod data, with the lower part of all supermodules. The neutrino-induced events collected with these partial configurations have been analyzed and the results published [14].

Most of the results presented here are based on data collected with the full configuration of the detector $(6 \mathrm{su}-$ permodules with lower part and attico) between April 29, 1994 and December 20, 2000 when the acquisition was stopped. During this period more than 40 million downgoing muon events were recorded. The duty-cycle of the data-taking was $84 \%$ ( $97 \%$ for the supernova watching system [28]). The effective live-time was 5.57 years with the full detector, 0.46 years for 6 -smod and 1.38 years for 1 -smod data-taking (details about the effective live-time estimates are in Appendix A).

\section{Physics and detector simulation}

The simulation of atmospheric neutrino events required the development of physics generators based on atmospheric neutrino fluxes and neutrino cross sections.

In order to understand the most suitable inputs to the physics generator it is important to consider the current state of calculations of atmospheric neutrino fluxes.

Even though many aspects of these calculations have been further developed recently, the absolute normalization is still uncertain and this uncertainty increases with energy $[29,30]$. Comparing different atmospheric neutrino calculations, it can be estimated that the absolute normalization of $\nu$-fluxes is still affected by $15 \%$ and $30 \%$ errors in the $\lesssim 10 \mathrm{GeV}$ and $\gtrsim 100 \mathrm{GeV}$ energy ranges respectively. This uncertainty is large even if calculations are benchmarked against atmospheric muons at sea level and in the atmosphere. The main errors come from the primary cosmic ray $(\mathrm{CR})$ spectrum and the hadronic interaction models.

During the last few years, authors of different Monte Carlo computations compared their interaction models and this led to code updates. The generator TARGET used in past calculations [31] was updated by the Bartol group [32]. Other groups used more sophisticated interaction and transport codes, such as FLUKA [33,34], extensively benchmarked on accelerator data and muons. The Japanese group adopted the DPMJET3 interaction model $[35,36]$.

The other important input is the primary CR spectrum whose uncertainties are energy dependent. The fit presented at ICRC2001 [37] was an attempt to unify different calculations. At energies $<200 \mathrm{GeV} /$ nucleon it relies on recent measurements [38,39]: for protons these measurements are in agreement within $5 \%$, while for $\mathrm{He}$ there is a discrepancy at the level of $15 \%$. At high energy $\left(\gtrsim 10^{4} \mathrm{GeV} /\right.$ nucleon $)$ the JACEE [40] and RUNJOB [41] data have large errors leaving room for large variations in the fitted spectral slope $(\gamma)$. While at energies $\lesssim 100 \mathrm{GeV} /$ nucleon, the ICRC2001 fit [37] changed by less than 10\% compared to the previous Bartol 96 fit [31], at higher energies the ICRC2001 fit is steeper $(\gamma \simeq-2.74)$ than the Bartol 96 fit $(\gamma \simeq-2.71)$.

The effect on $\nu$-fluxes of changing the CR flux slope above $100 \mathrm{GeV} /$ nucleon has been investigated in [34,36]. When the FLUKA interaction code is used with the ICRC 2001 fit in the energy range of interest for our experiment, it produces a $\nu_{\mu}$ flux lower than that obtained with the Bartol 96 fit. The difference is $5 \%$ at $10 \mathrm{GeV}$ and $20-30 \%$ between $100 \mathrm{GeV}-1 \mathrm{TeV}$. On the other hand, if the same CR spectrum is used as input to the FLUKA and Japanese calculations the difference in the $\nu_{\mu}$ flux is within $10 \%$ [34], indicating a reasonable agreement in their atmospheric cascade modeling.

In order to minimize the impact of the atmospheric neutrino flux uncertainty on the oscillation parameter measurement, in this paper we have chosen to use quantities known at the level of $\sim 5 \%$, such as the distributions of zenith angles (discussed in Sect. 5.2) and ratios where most of the errors cancel, e.g. vertical-horizontal and low-high energy (see Sect. 7). Hence the presented results are independent of the absolute normalization of the flux and other $\nu$-flux models produce only small differences.

The FLUKA [33] atmospheric neutrino flux, with the ICRC2001 fit [37], was used at low energy. This choice was made because of the completeness of the code and of the agreement between the new measurements of the low energy primary CR spectrum. For the UpThrough events, we used the Bartol flux [31]. Nevertheless, some comparisons with other flux calculations are presented in Sect. 5.2. We have checked that, to within 5\%, FLUKA and Bartol calculations give the same predictions for the ratios quoted above.

Different neutrino event generators were used and the interactions were simulated both inside the detector and in the surrounding rock. The details of the physical gen- 
Table 1. Comparison of the A and B analyses for UpThrough events. The expected events are calculated assuming the Bartol $\nu$-flux [31]. The results of the last column refer to the analysis A applied to the full sample of MACRO data (1-smod, 6-smod and attico data). $P_{0}$ is the one-sided probability that the measurement is compatible with the predicition without oscillations; $P_{\tau}$ is the two-sided probability assuming oscillations. The uncertainty on the $\nu$-flux normalization is not taken into account

\begin{tabular}{lccc}
\hline & Analysis A & Analysis B & Analysis A (full sample) \\
\hline Observed events & 821 & 870 & 902 \\
Incorrect $1 / \beta$ (fit) & -26 & -43 & -31 \\
Soft Pions & -13 & -13 & -14 \\
Final number of events & 782 & 814 & 857 \\
Expected events with no oscillation & 1062 & 1125 & 1169 \\
$P_{0}$ & $1.3 \times 10^{-6}$ & $1.4 \times 10^{-7}$ & $4.3 \times 10^{-7}$ \\
Expected events assuming oscillation & 746 & 798 & 820 \\
$P_{\tau}$ & 0.49 & 0.77 & 0.51 \\
Median $\nu$-energy $(\mathrm{GeV})$ & & & $\sim 50$ \\
\hline
\end{tabular}

erators will be discussed below in relation to the different neutrino subsamples. The energy loss for muons propagating through rock was taken from [42], adjusting the energy loss for the chemical composition of the Gran Sasso rock.

A MC program (GMACRO) based on the GEANT package [43] was used to simulate the response of the detector. The ST response was simulated by introducing the processes which affect the distributions of cluster widths (induced charge distribution on the pick-up strips, electronics performance, delta-ray production and so on). The signal from a particle traversing a scintillation counter was approximated by assuming that the energy loss occurred in the middle position between entry and exit points at the average of entry and exit times. All parameters of streamer tubes and scintillation counters were set in order to reproduce the average performances of the reference runs (see Appendix A).

The output buffers produced by GMACRO were identical in format to those produced by the acquisition system so that real and simulated data were studied with the same fully automatic analysis chain (raw data - data summary tapes - analysis output). Only in the InDown+UpStop analysis is there need for a visual scanning of data that is performed in the same way for real and simulated data.

\section{High energy neutrinos}

The UpThrough topology is very clear in MACRO. The atmospheric muons are not rejected at trigger level as in some other experiments. The tracks with $1 / \beta$ in the signal range $[-1.25,-0.75]$ are accepted as upward-going at the end of the analysis chain. The up-down symmetry of the detector and of the analysis chain allows us to keep the downwardgoing muons together with the upward-going sample, which is smaller by about a factor of $10^{-6}$. This allows us to study the detector systematics, the efficiency of the analysis cuts, the acceptance, and the accuracy of the simulation using the large sample of atmospheric downward-going muons.

Two independent analyses were developed with different approaches: the analysis labeled A starts from a muon track reconstructed by the ST system and looks for at least two scintillator hits geometrically compatible with the track. The analysis labeled B starts from all pairs of scintillator hits and looks for a ST track consistent with each pair. In both analyses the zenith angular information is obtained from the ST track.

The two analyses are different in many other respects (they are fully described in Appendix B), but the results are in full agreement.

Two main background sources were identified. The first is a physical background from upward-going particles, mainly pions, produced by undetected atmospheric muons [44]. To reduce this background, the analyses require that the track crosses at least $200 \mathrm{~g} / \mathrm{cm}^{2}$ of the rock absorber in the lower part of MACRO (ABS cut). On the basis of our measurement we estimate [44] that 2.3 events per year survive this cut (the subtraction of this background is labeled "Soft Pions" in Table 1).

The second principal background is from atmospheric horizontal muons crossing the thinner layer of rock in the direction of the Teramo valley. Because of multiple-scattering, some horizontal muons will appear as upward-going. The two analyses apply a slightly different azimuthal angular cut (Teramo cut) to remove the events from the Teramo direction, for events with $|\cos \theta|<0.1$.

Other contaminations of the $1 / \beta$ distribution come from muon decays in which the electron hits a scintillation counter; these can produce large positive values of $1 / \beta$ because of the decay time. Incorrect $1 / \beta$ values happen most frequently, however, in events with multiple muons or showers; these can cause more than one track to cross a scintillator yielding an incorrect time measurement. This contamination gives a contribution that is approximately flat in $1 / \beta$ in the region of interest and can be estimated and corrected from the observed distribution.

As a further check, a third analysis was performed with completely independent trigger and electronics (see Appendix C). It was consistent with the two analyses just discussed.

The simulation of the expected signal for the Up Through sample is discussed in detail in Sect. 5.2. Unless explicitly 
Table 2. Detected and expected (Bartol) events for the analysis A applied to the complete sample in different zenith angle bins. The assumed oscillation is $\nu_{\mu} \rightarrow \nu_{\tau}$ with maximal mixing and $\Delta m^{2}=0.0023 \mathrm{eV}^{2}$

\begin{tabular}{lcccc}
\hline $\cos \theta$ & Detected events & Systematical error $(\%)$ & Expected events & Expected events with oscillation \\
\hline-0.95 & 110.0 & 6 & 188.4 & 117.5 \\
-0.85 & 93.6 & 6 & 173.8 & 111.4 \\
-0.75 & 113.9 & 6 & 160.2 & 105.4 \\
-0.65 & 122.6 & 6 & 146.6 & 100.1 \\
-0.55 & 91.7 & 6 & 134.1 & 94.0 \\
-0.45 & 95.5 & 6 & 118.0 & 86.7 \\
-0.35 & 89.7 & 6 & 102.9 & 79.6 \\
-0.25 & 76.3 & 6 & 79.4 & 65.9 \\
-0.15 & 47.3 & 6 & 52.5 & 47.1 \\
-0.05 & 16.4 & 10 & 12.9 & 12.3 \\
\hline
\end{tabular}

stated otherwise, we will always assume the Bartol neutrino flux [31] in the following.

\subsection{Comparison of analyses A and B}

Analyses A and B give very nearly the same results. As shown in Fig. 15, there is a difference of $5.8 \%$ in the number of events (including down-going trajectories) in the two analyses. This arises from the fact that in analysis B only one entry is selected for an event while in analysis A, more entries are accepted. Thus the contribution of multiple muon events is different in the two analyses.

There are 754 Up Through events common to both analyses. A further 67 events are accepted by analysis A only and 116 by analysis B only, but the background estimated by means of the fit procedure is higher for analysis $\mathrm{B}$. The presence of events in one analysis and not in the other is well explained by the different analysis choices and was expected; the differences are well reproduced in the analysis of simulated data. The compatibility of the two analyses is evident from Fig. 3 in which analysis A is also applied to data taken when the apparatus was incomplete.

\subsection{Comparison between data and simulation}

The GMACRO program has been interfaced with a generator of neutrino-induced muons based on the Bartol neutrino flux [31] and on the GRV94 [45] parton distributions for calculation of the $\nu N$ cross section. Both analyses introduce correction factors to the expected signal that take into account tiny differences between real and simulated data (for instance the effect of the micro-cuts which exclude some badly performing scintillation counters or modules).

The upward-throughgoing muon sample includes a small contribution of internal interaction events with the vertex close to the bottom scintillator layer with an upwardgoing muon energetic enough to reach another scintillator layer. They are properly InUp events but it is impossible to distinguish them from the UpThrough sample. To estimate this contribution we have used the generator described in Sect. 6.3.

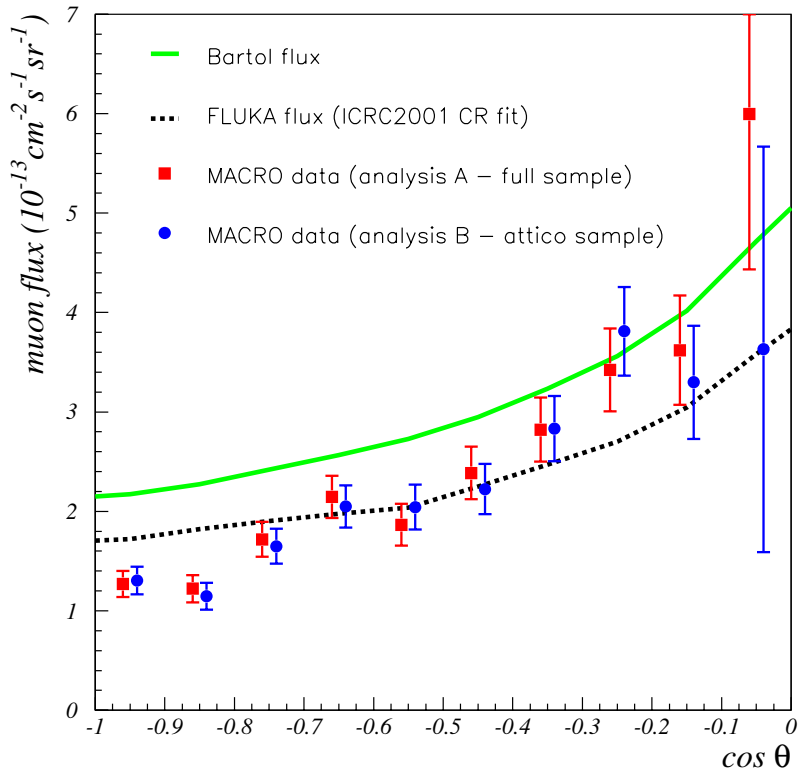

Fig. 3. Comparison of the UpThrough muon fluxes measured by means of the different analysis procedures, A and B. The experimental points are slightly shifted horizontally to distinguish the two analyses. Statistical and systematic errors are displayed. The non-oscillated Bartol [31] and FLUKA [34] fluxes, assuming $E_{\mu}>1 \mathrm{GeV}$, are shown (the theoretical error is not displayed). The fit to the new CR measurements [37] is used for the FLUKA flux

The shape of the zenith distribution is much better known (5\%) than the absolute normalization of the upgoing muon flux $(\sim 25 \%)$, as discussed in Sect. 4 .

This is a consequence of the fact that the angular distribution is nearly isotropic and is determined by simple geometrical effects [46]. The main source of systematic error is the uncertainty in the kaon/pion ratio $(\sim 3 \%)$. As a matter of fact neutrinos from kaon decay are approximately isotropic, since nearly all kaons decay, while pions have longer lifetime. Vertical pions have a large probability of interacting before decay, and the horizontal/vertical ratio is enhanced. An additional source of uncertainty is the shape of the energy spectrum, since the $\nu$-interaction 


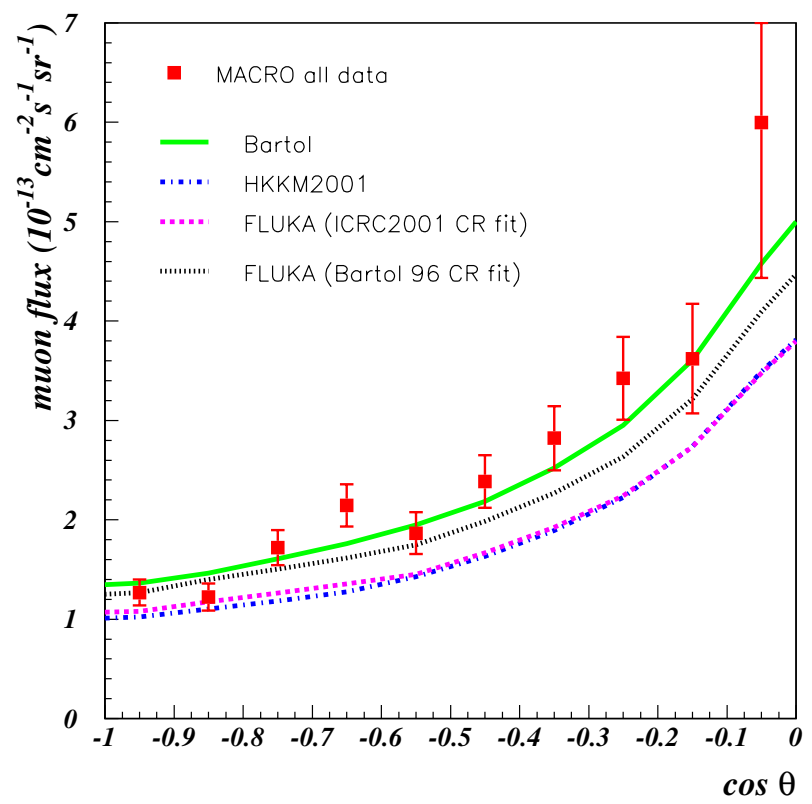

Fig. 4. Comparison between the measured angular distribution of the UpThrough muon flux and the MC predictions assuming $\nu$-oscillations with the MACRO parameters. The solid curve indicates the Bartol flux [31], the dashed curve is for the FLUKA flux [34] fitted to the new CR measurements [37], the dotted curve is again the FLUKA flux with the fit in [31], and the dash-dotted line is for the HKKM-2001 flux [35]

cross-section is energy dependent. This produces an uncertainty of $\sim 2 \%$. Finally another $1.3 \%$ uncertainty comes from seasonal effects and our ignoring the latitude dependence of the atmosphere's density profile. Adding all these contributions in quadrature we estimate the theoretical uncertainty on the shape to be about $4 \%$.

In order to verify that using different calculations affects the zenith distribution at the level of a few percent (while it has an effect of the order of $\sim 25 \%$ on the event rates) we used, besides the Bartol flux [31], the FLUKA [34] and HKKM-2001 [35] calculations. In the MACRO detector the FLUKA (with the ICRC2001 fit) atmospheric $\nu$-flux produces an event rate $\sim 23 \%$ lower than the Bartol flux, while the shapes of the angular distributions differ not more than $6 \%$. The HKKM-2001 calculation gives event rates that are about 25\% lower than Bartol, but the shapes of the distributions differ by less than $4 \%$. The FLUKA and HKKM-2001 angular distributions differ in shape by less than $6 \%$.

The new calculations HKKM-2001 and FLUKA show good agreement when they use the same fit to the primary CR flux [37], confirming the improvement in the hadronic model. In Fig. 4 our data are compared with the oscillated Bartol, HKKM-2001 and FLUKA calculations using the new CR fit. All predicted curves are for maximal mixing and $\Delta m^{2}=0.0023 \mathrm{eV}^{2}$. It is clear from the figure that the recent fit to the primary $\mathrm{CR}$ energy distribution yields a neutrino flux that is too low to account for our measurement of upward-throughgoing muons.

In Fig. 5 the ratio of the number of detected UpThrough events to the number of expected events is displayed as a

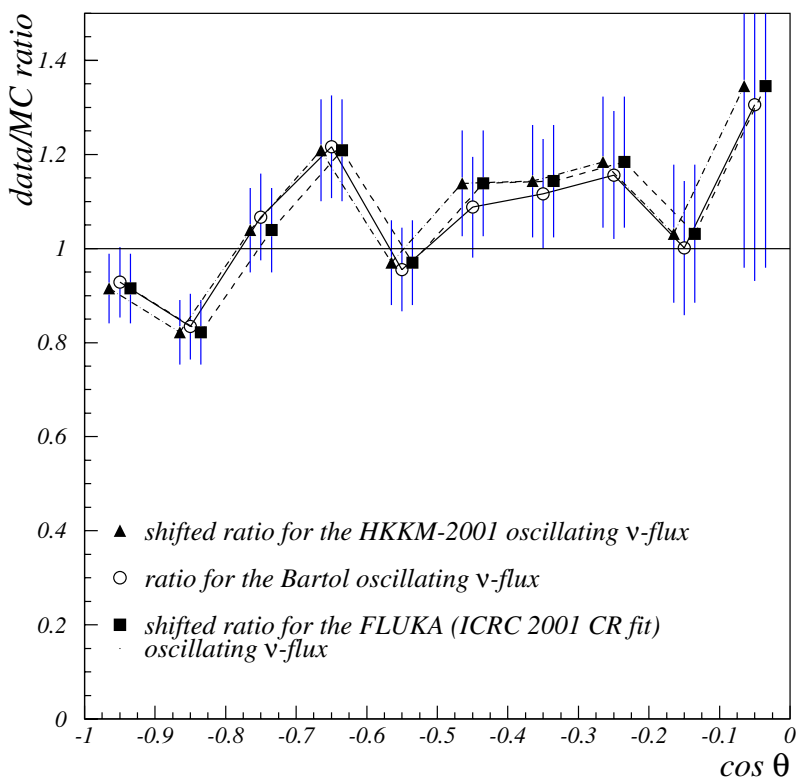

Fig. 5. Ratio of the detected UpThrough events to the expected ones, assuming $\nu_{\mu} \rightarrow \nu_{\tau}$ oscillation with maximal mixing and $\Delta m^{2}=0.0023 \mathrm{eV}^{2}$. The neutrino fluxes used are the Bartol flux [31], the FLUKA flux [34] fitted to the new CR measurements [37] and the HKKM-2001 flux [35]. The points are shifted slightly horizontally in order to distinguish the fluxes. In order to ease the shape comparison the ratios for the FLUKA and HKKM-2001 fluxes are properly reduced. Statistical and systematic errors are displayed

function of the zenith angle, assuming different oscillated $\nu$-fluxes. The FLUKA and HKKM-2001 expected rates are arbitrarily translated to make the shape comparison easier. The shape of the distribution is modified only at the level of a few percent by the assumption of different $\nu$-fluxes.

\subsection{Checks and systematics study}

Several checks were made to look for detector systematics in the analysis. The data sample was split into 4 subsamples with equal live-time and the angular distributions were compared with the Kolmogorov test. The four resulting distributions are statistically compatible (the lowest compatibility is $13 \%$, and the highest is $71 \%$ ).

The azimuthal distributions of Fig. 6 confirm the agreement of the two analyses. The fit of these distributions to a constant demonstrates that the detector acceptance is well reproduced for each azimuth value. A uniform deficit independence of the azimuth angle is the expected effect for $\nu$-oscillation.

In Fig. 7 the events are displayed with respect to months and hours. Both distributions are consistent with being constant. An apparent modulation is present in the month distribution, but it is not statistically significant. We can exclude detector systematic uncontrolled effects because we were able to observe an atmospheric temperature-induced modulation smaller than this in the downgoing flux [47].

The dependence of the measurement on the efficiency of the scintillator counters and the ST system has been studied 


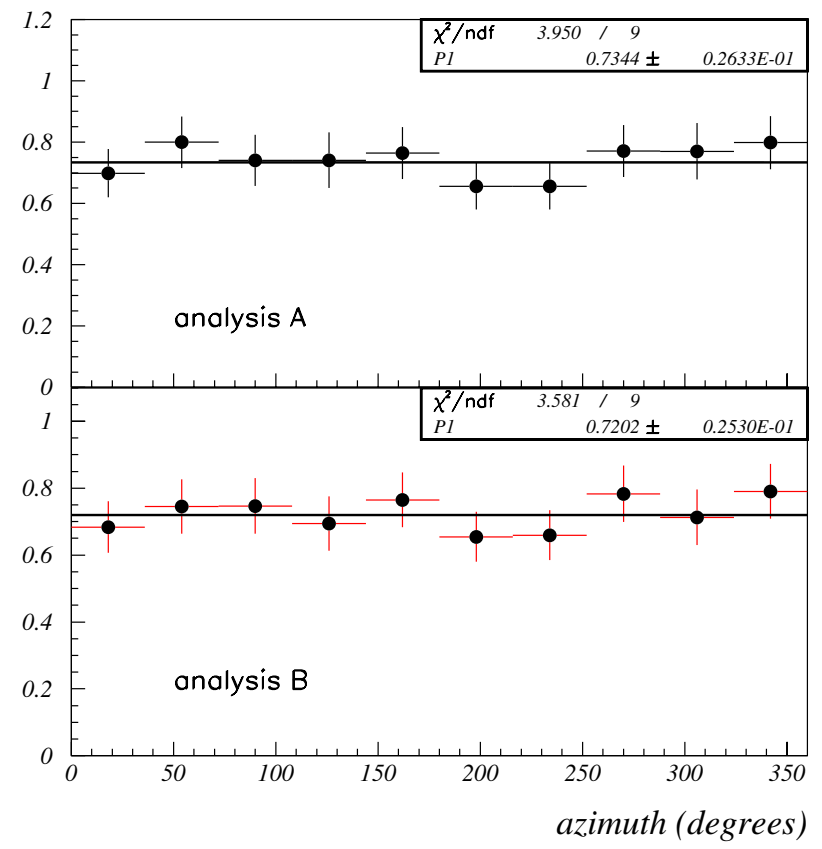

Fig. 6. Ratio of detected UpThrough events to the number expected without $\nu$-oscillation as a function of azimuth angle. Only statistical errors are displayed. The line is a fit to a constant
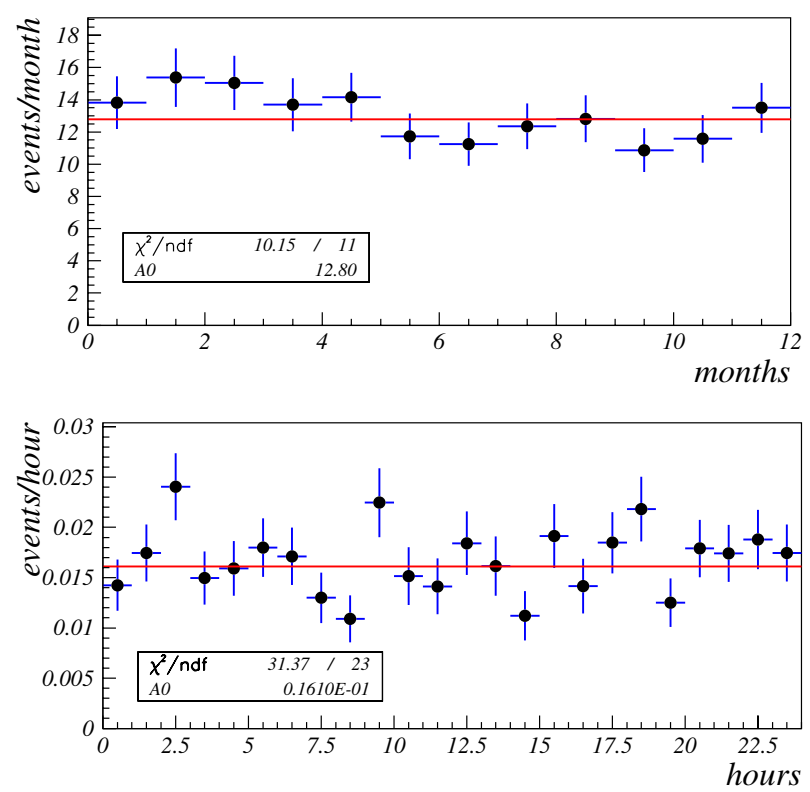

Fig. 7. UpThrough event rate normalized to the live-time vs months (first plot) and vs hours (second plot) in the analysis B. Only statistical errors are displayed. The constant fit is superimposed

by simulations and by introducing artificial inefficiencies into the real data set. The two methods for estimating efficiency are in agreement.

The systematic uncertainties have been estimated by applying the same analysis procedure (without the requirement $1 / \beta<0)$ to the large sample of downward-going muons. Real and simulated data are in good agreement and the systematic errors are estimated from the differences.

\subsection{Muon energy estimate}

An estimate of the energy of upward-throughgoing muons provides an important contribution to the understanding of the oscillation mechanism. Although a significant fraction of the neutrino energy is absorbed by the hadrons in the neutrino interaction and subsequently lost during the muon propagation to the detector, MC simulations show a linear correlation between the parent neutrino energy $E_{\nu}$ and the muon residual energy $E_{\mu}$ at the detector.

In MACRO the only way to estimate the muon energy is to measure Multiple Coulomb Scattering in the detector's rock absorber with the ST system. This is described in detail in $[20,21]$. It is based on the relation between the $R M S$ of the lateral displacement and the momentum of relativistic particles crossing a layer of material. A first analysis was performed using the digital information from the streamer tubes [21] with an intrinsic spatial resolution $\sigma_{x} \simeq 1 \mathrm{~cm}$. This analysis showed the feasibility of the method for muon energies up to $\sim 10 \mathrm{GeV}$. To improve the spatial resolution to $\sigma_{x} \simeq 3 \mathrm{~mm}$, the drift time in the streamer tubes was measured by using the QTP-TDC system developed for magnetic monopole searches [48]. An absolute muon energy calibration was performed in the CERN PS-T9/SPS-X7 beam using a Neural Network to handle the scattering variables and to reconstruct the muon energies. The global neutrino energy resolution is $\sim 150 \%$.

Zenith angle and neutrino energy have been reconstructed for each event to obtain the ratio $L_{\nu} / E_{\nu}$. The result is shown in Fig. 8, where an additional point coming from the InUp sample (see Sect. 6.1) has been added.

To quantify the independent sensitivity of this analysis to neutrino oscillations, a MC was used to define the best parameter to separate the oscillation from the nooscillation hypothesis. The ratio $N_{\text {low }} / N_{\text {high }}$ gives the best performance, where $N_{\text {low }}\left(N_{\text {high }}\right)$ is the number of events with $E_{\nu}<30 \mathrm{GeV}\left(E_{\nu}>130 \mathrm{GeV}\right)$. The measured ratio

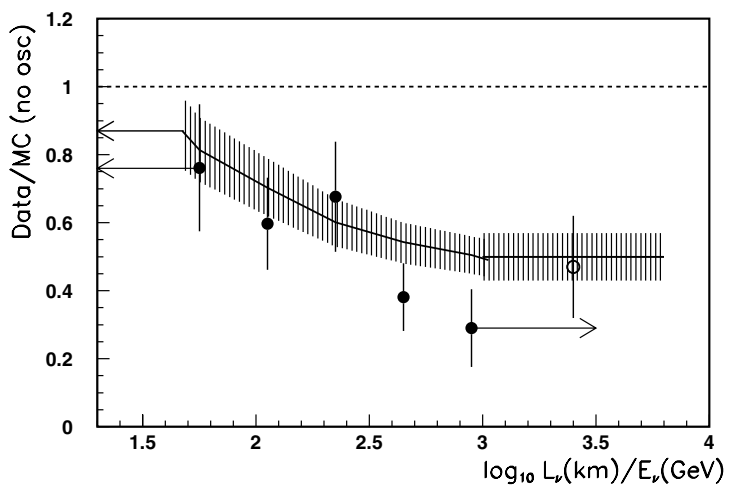

Fig. 8. Ratio data/MC (no oscillations) as a function of the estimated $L_{\nu} / E_{\nu}$ for the UpThrough muon sample (black points). The solid line is the MC expectation assuming $\Delta m^{2}=$ $0.0023 \mathrm{eV}^{2}$ and $\sin ^{2} 2 \theta_{m}=1$. The last point (empty circle) is obtained from the $\operatorname{In} U p$ sample 
is $R_{\text {meas }}=0.85 \pm 0.16_{\text {stat }}$; the $\mathrm{MC}$ prediction in case of no oscillations is $R_{0}=1.50 \pm 0.25_{\text {theo+sys }}$, while for $\Delta m^{2}=$ $0.0023 \mathrm{eV}^{2}$ and $\sin ^{2} 2 \theta_{m}=1, R_{\tau}=1.00 \pm 0.17_{\text {theo+sys }}$ (see Table 4).

\section{Low energy neutrinos}

Two event subsamples (InUp and UpStop+InDown) are generated by low energy atmospheric neutrinos. They are studied by means of different analysis methods discussed below (more details in [18]).

\subsection{InUp events}

The identification of $\operatorname{In} U p$ events is based on topological criteria and ToF measurements. The basic requirement is the presence of at least two scintillator clusters in the upper part of the apparatus (see Fig. 1) matching a ST track reconstructed in two views.

For In $U p$ candidates, the lower end of the track must be inside the apparatus. To reject fake semi-contained events entering through a detector crack, the extrapolation of the track in the lower part of the detector must cross and not fire at least one horizontal streamer tube plane and one bottom scintillation counter or three streamer tube planes and one scintillation counter on lateral walls.

The above conditions are based on the study of simulated events and take into account the detector inefficiencies. Therefore the contribution from upward-throughgoing muons which mimic semi-contained muons is reduced to less than $\sim 1 \%$. The measured $1 / \beta$ distribution is shown in Fig. 9. The $1 / \beta$ range $[-1.3,-0.7]$ is choosen to define the signal on the basis of MC simulation. A total of 164 events survive in this range after background subtraction.

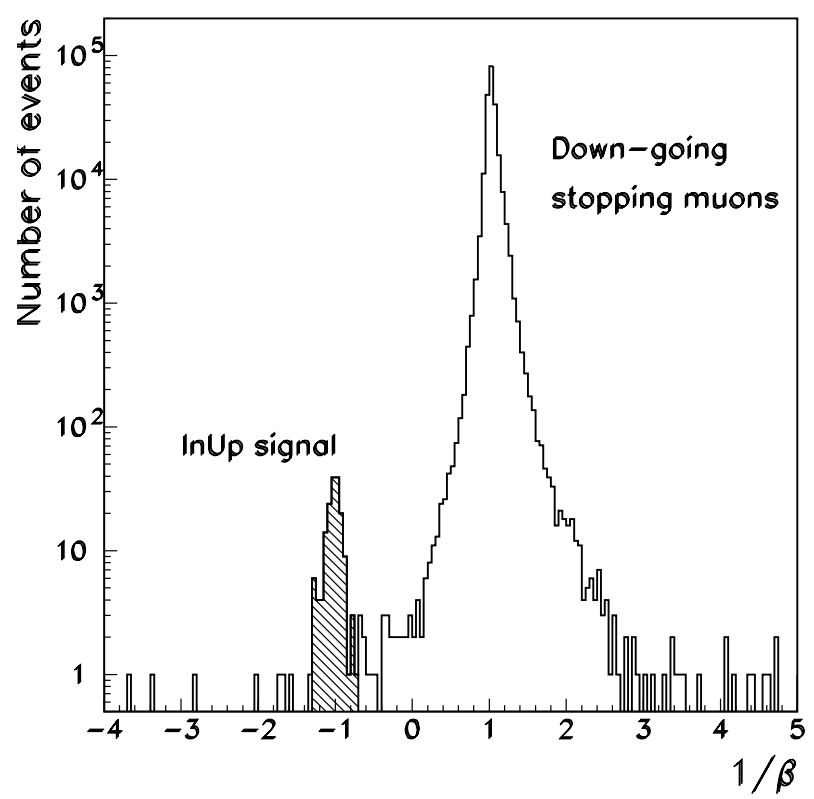

Fig. 9. The $1 / \beta$ distribution of partially-contained events. The peak at $1 / \beta \sim+1$ is due to downward-going muons stopping in the apparatus

\subsection{UpStop+InDown events}

The identification of UpStop +InDown events is based on topological criteria. The candidates have a track starting (ending) in the lower apparatus, and crossing the bottom detector face. The track must also be located or oriented in such a way that it could not have entered (exited) undetected through insensitive zones in the apparatus.

The event selection requires the presence of one reconstructed track crossing the bottom layer of the scintillation counters (see Fig. 1). The event vertex (or $\mu$ stop point) in the detector is identified in the same way as for the InUp search, with the additional requirement that all hits along the track must be at least $1 \mathrm{~m}$ inside each supermodule. To reject ambiguous and/or wrongly tracked events surviving the selection, a scan with the Event Display was performed. The main background comes from upward-going charged pions which are estimated to contribute 10 events to this analysis [44]. After the full analysis chain and the background subtraction, 262 events were classified as $U p$ Stop+InDown events; see Table 3.

\subsection{Comparison between data and Monte Carlo}

The expected rates were evaluated with a full MC simulation. The events are mainly due to $\nu_{\mu} \mathrm{CC}$ interactions, with small contributions from neutral current (NC) and $\nu_{e}$ interactions $(\sim 13 \%$ for InUp and $\sim 10 \%$ for UpStop + InDown $)$ in a no-oscillation scenario. An almost equal number of UpStop and InDown events are expected in our data sample. The $\nu_{e}$ and $\nu_{\mu}$ were allowed to interact in a volume containing the experimental hall with the detector. The rock mass in the generation volume was $169.6 \mathrm{kton}$, while the MACRO mass was $5.3 \mathrm{kton}$. We used the atmospheric $\nu$-flux calculated by the FLUKA code [33] at the solar cycle average and the neutrino cross sections from [49]. The number of expected events was also evaluated using the NEUGEN neutrino interaction generator [50] which gives $\sim 5 \%$ fewer events than our $\nu$-interaction generator.

Most $(\sim 90 \%)$ of MACRO's partially contained and stopping events are induced by atmospheric neutrinos with energies smaller than $10 \mathrm{GeV}$ (see Fig. 2); the energies of the corresponding primary CRs are well below $100 \mathrm{GeV}$, and those primaries are mainly protons and $\mathrm{He}$ nuclei. As discussed in Sect. 4, in this energy interval the primary spectrum was recently measured by BESS [39] and AMS [38] with a systematic uncertainty less than 5\%. According to [33], the overall theoretical uncertainty on modulation, geomagnetic cutoff treatment and hadronic interaction model is $20 \%$. Another $5 \%$ uncertainty comes from cross sections. The total theoretical uncertainty is $21 \%$.

The systematic uncertainty in the detector response, arising from the uncertainty in the detector mass (5\%), the acceptance vs zenith angle $(5 \%)$, the live-time estimate $(3 \%)$, the uncertainty in the effective containment of the interaction vertex $(4 \%)$, the fluctuations of the detector efficiency $(2 \%)$, the scanning efficiency for the UpStop+InDown (2\%) and GEANT physical parameters $(1 \%)$, and from the uncertainty in the subtraction of the 
Table 3. Summary of low-energy neutrino analyses. The estimated background has been subtracted from the event numbers. The oscillation parameters estimated in Sect. 7.1 are assumed for the $\nu$-oscillation

\begin{tabular}{lcc}
\hline & InUp & InDown+UpStop \\
\hline Observed events & $157 \pm 12_{\text {stat }}$ & $262 \pm 16_{\text {stat }}$ \\
Expected events & $235 \pm 26_{\text {sys }} \pm 48_{\text {theo }}$ & $315 \pm 34_{\text {sys }} \pm 64_{\text {theo }}$ \\
Assuming oscillation & $135 \pm 15_{\text {sys }} \pm 27_{\text {theo }}$ & $238 \pm 26_{\text {sys }} \pm 48_{\text {theo }}$ \\
\hline Median $\nu$-energy $(\mathrm{GeV})$ & 2.4 & 2.2 \\
\hline
\end{tabular}

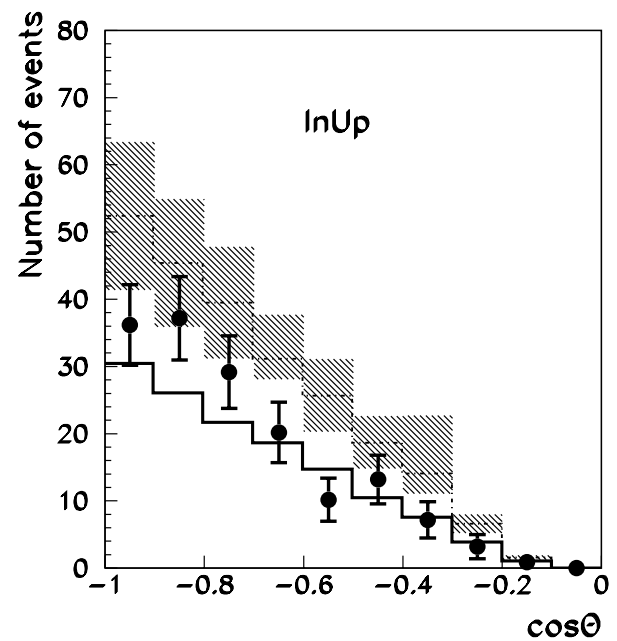

background (4\%), is about $9 \%$ when all the contributions are added in quadrature.

Table 3 gives the numbers of observed and expected events for the low energy neutrino samples. In Fig. 10 the measured angular distributions are compared with the MC predictions.

The measured number of InUp events is about $67 \%$ of the value expected in the case of no $\nu_{\mu}$ oscillations $(54 \%$ assuming the Bartol $\nu$-flux [31]). Combining the statistical, systematic and theoretical errors, the one-tailed Gaussian probability of this measurement is $8 \%$.

If the observed deficits were due to a theoretical overestimate of the neutrino fluxes and/or cross sections, one would expect the same reduction for the sample of $U p$ Stop + InDown events. These events are induced by parent neutrinos with almost the same energy spectrum. We evaluated a residual $5 \%$ theoretical error in the ratio between the expected number of InUp and UpStop + InDown events due to small differences of the energy spectra and geomagnetic effects. The systematic uncertainty on the ratio is reduced to $\sim 6 \%$ because of cancellations. The measured ratio $I n U p /(U p S t o p+I n D o w n)$ is $R_{\text {meas }}=0.60 \pm 0.06_{\text {stat }}$, while the expected value is $R_{0}=0.745 \pm 0.06_{\text {theo+sys }}$ for no oscillations. The probability to obtain a ratio this far or farther from the expected is $4.3 \%$, taking into account the non-Gaussian shape of the uncertainty on the ratio. This probability is almost independent of the assumptions about neutrino fluxes and neutrino cross sections.

The two data sets are consistent with neutrino oscillations $\left(\nu_{\mu}\right.$ disappearance) with maximal mixing and $\Delta m^{2} \sim 0.001 \div 0.01 \mathrm{eV}^{2}$. Upgoing muon neutrinos which induce InUp and UpStop events are reduced by $50 \%$. No reduction is expected for InDown events. As a rough prediction, we expect a rate reduced by $50 \%$ for $I n U p$ and by $25 \%$ for UpStop + InDown events.

Using the oscillation parameters obtained in Sect. 7.1, the expected angular distributions (Fig. 10) and the total numbers (Table 3 ) are in agreement with the measurement.

\section{Physical models and experimental results}

Many $\nu$-oscillation mechanisms have been suggested to explain the atmospheric neutrino data. Here only two flavor mechanisms are examined; we assume that the contribution of channels involving $\nu_{e}$ is negligible for the energies and path-lengths of interest [51].

\section{$7.1 \nu_{\mu} \rightarrow \nu_{\tau}$ oscillations}

The uncertainty on the absolute value of the neutrino fluxes is large for the energies of interest [29]. However most of the uncertainty cancels in ratios between numbers of events belonging to different categories. To test the no-oscillation hypothesis and to evaluate the sensitivity, we considered the following three ratios :

1. the ratio (Vertical/Horizontal) between events with $\cos \theta \leq-0.7$, and events with $\cos \theta \geq-0.4$ in the high energy sample. This is the best ratio of counts in different angular ranges for discriminating between the 
Table 4. Ratios between different event categories: $R_{\text {meas }}$ is the measured value; $R_{\tau}$ is the theoretical value expected for $\nu_{\mu} \rightarrow \nu_{\tau}$ oscillations with $\Delta \mathrm{m}^{2}=2.3 \times 10^{-3} \mathrm{eV}$ and maximal mixing; $R_{0}$ is the expected value for no oscillations. As indicated, the last two columns give the probability of an experiment observing a ratio $R$ equal to or smaller than our measurement assuming there are no oscillations. The distribution of $R$ depends upon $R_{0}$, its theoretical and experimental systematic uncertainty $\sigma_{0}$, and the number of events observed, $N$. We allocate the systematic errors to the numerator and denominator of $R$ so they make equal contributions to the uncertainty in the ratio. Further, we choose two different, fixed possible values for $N$, as indicated

\begin{tabular}{lcccccc}
\hline Category & $N_{\text {detected }}$ & $R_{\text {meas }} \pm \sigma_{\text {stat }}$ & $R_{\tau}$ & $R_{0} \pm \sigma_{0}$ & \multicolumn{2}{c}{$P\left(R \leq R_{\text {meas }} \mid R_{0}, \sigma_{0}, N\right)$} \\
& & & & & $N=N_{\text {detected }}$ & $N=N_{\text {no osc }}$ \\
\hline Vertical/Horizontal & 547.3 & $1.38 \pm 0.12$ & 1.61 & $2.11 \pm 0.13$ & $4.5 \times 10^{-5}$ & $6.4 \times 10^{-6}$ \\
$N_{\text {low }} / N_{\text {high }}$ & 100.5 & $0.85 \pm 0.16$ & 1.00 & $1.50 \pm 0.25$ & $1.9 \times 10^{-2}$ & $7.7 \times 10^{-3}$ \\
InUp/(InDown+UpStop $)$ & 418.4 & $0.60 \pm 0.06$ & 0.56 & $0.745 \pm 0.06$ & $4.3 \times 10^{-2}$ & $3.1 \times 10^{-2}$ \\
Combination & & & & & $6 \times 10^{-6}$ & $3.4 \times 10^{-7}$ \\
\hline
\end{tabular}

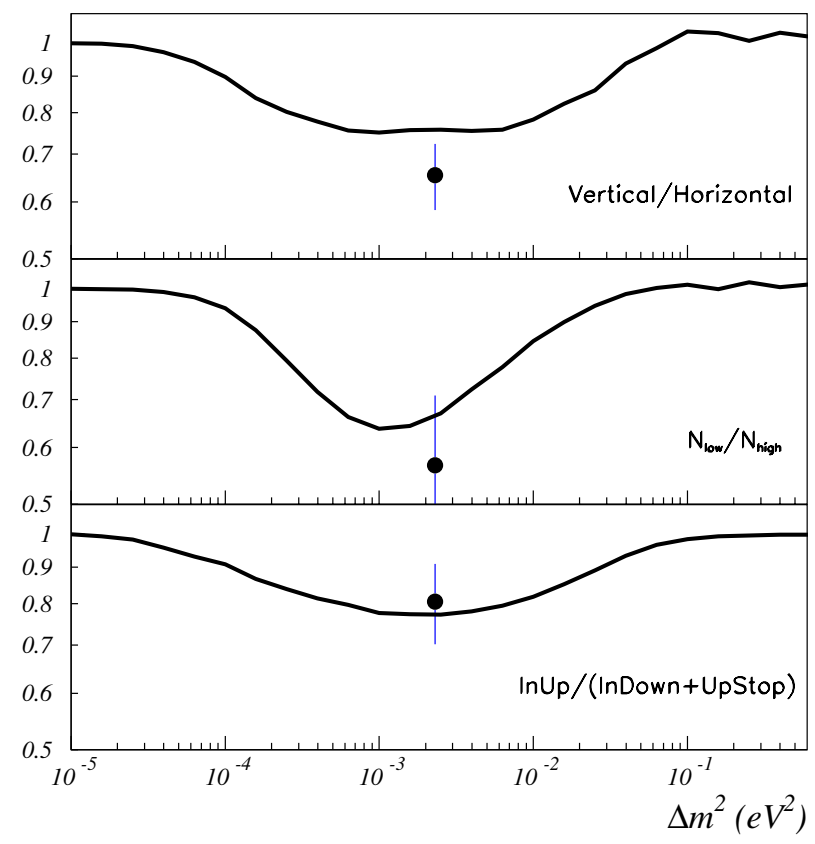

Fig. 11. The ratios, described in the text, as a function of $\Delta m^{2}$ with maximal mixing, normalized to obtain 1 for no oscillations. The measurement significances depend not only on these ratios, but also on the statistical and systematical errors. The points are the experimental data inserted at $\Delta m^{2}=0.0023 \mathrm{eV}^{2}$. For a discussion of systematical uncertainties see [19]

no-oscillation and the $\nu_{\mu} \rightarrow \nu_{\tau}$ oscillation hypotheses [19]. The error affecting this ratio is about $6 \%$, obtained by combining the systematic error on the acceptance of $4.6 \%$ and theoretical errors of $4 \%$;

2 . the ratio between events in the sub-categories $\left(N_{\text {low }}\right.$ and $N_{\text {high }}$ ) as defined in Sect. 5.4. The combined error in this ratio is about $17 \%$. This large uncertainty arises mainly from the primary CR spectrum;

3 . the ratio between events in the low energy categories InUp and InDown + UpStop. The combined error on this ratio is about $6 \%$.

The first two ratios were obtained using MCs to find the estimators with the best performances in separating the oscillation and no-oscillation hypotheses. Fig. 11 shows the behavior of 3 ratios as a function of $\Delta m^{2}$ assuming maximal mixing. In principle the ratio $N_{\text {low }} / N_{\text {high }}$ has a better sensitivity to the $\Delta m^{2}$ value; however taking into account the detected number of events and the theoretical uncertainties, the ratio $I n U p /($ InDown $+U p S t o p)$ is the more statistically significant.

Table 4 shows the measured values of the three ratios and the predictions for no-oscillations. In the last two columns the corresponding probability values are given, obtained by allowing the predicted ratios to fluctuate around the mean values and taking into account the non-Gaussian distributions of the ratios. The fluctuations were introduced using either the observed number of events $(N=$ $\left.N_{\text {detected }}\right)$ or the predicted number without oscillations $\left(N=N_{\text {no osc }}\right)$.

Combining the three independent results [52], we obtain a probability of $6 \times 10^{-6}(\sim 5 \sigma)$. To evaluate the oscillation parameters we used a $\chi^{2}$-like function containing 3 terms:

$$
\begin{aligned}
\chi^{2}= & \sum_{i=1}^{10}\left(\frac{N_{i}^{(1)}-\alpha^{(1)} N_{i}^{(M C)}}{\sigma_{i}^{(1)}}\right)^{2} \\
& +\sum_{i=1}^{2}\left(\frac{N_{i}^{(2)}-\alpha^{(2)} N_{i}^{(M C)}}{\sigma_{i}^{(2)}}\right)^{2} \\
& +\sum_{i=1}^{2}\left(\frac{N_{i}^{(3)}-\alpha^{(3)} N_{i}^{(M C)}}{\sigma_{i}^{(3)}}\right)^{2}
\end{aligned}
$$

where the $\alpha$-parameters are constants introduced to normalize the predicted number of events to the data in each category. The first term is the 10-bin $\chi^{2}$ of the angular distribution of UpThrough events (analysis A for full sample). The second one is the 2-bin $\chi^{2}$ obtained using $N_{\text {low }}$ and $N_{\text {high }}$ events. The last term is the 2-bin $\chi^{2}$ obtained using the InDown+UpStop and InUp events.

To take into account the physical boundaries of the oscillation parameters the Feldman-Cousins procedure [53] was used. The result is shown in Fig. 12. We obtained $\chi^{2}=35.3$ for no oscillations. The highest probability $\left(\chi^{2}=\right.$ $10.05)$ is reached at $\Delta m^{2}=0.0023 \mathrm{eV}^{2}$ and $\sin ^{2} 2 \theta_{m}=1$. The $1 \sigma$ interval around this point on the $\Delta m^{2}$ axis is $[0.001,0.0065]$. The first and most important term of $\chi^{2}$ 


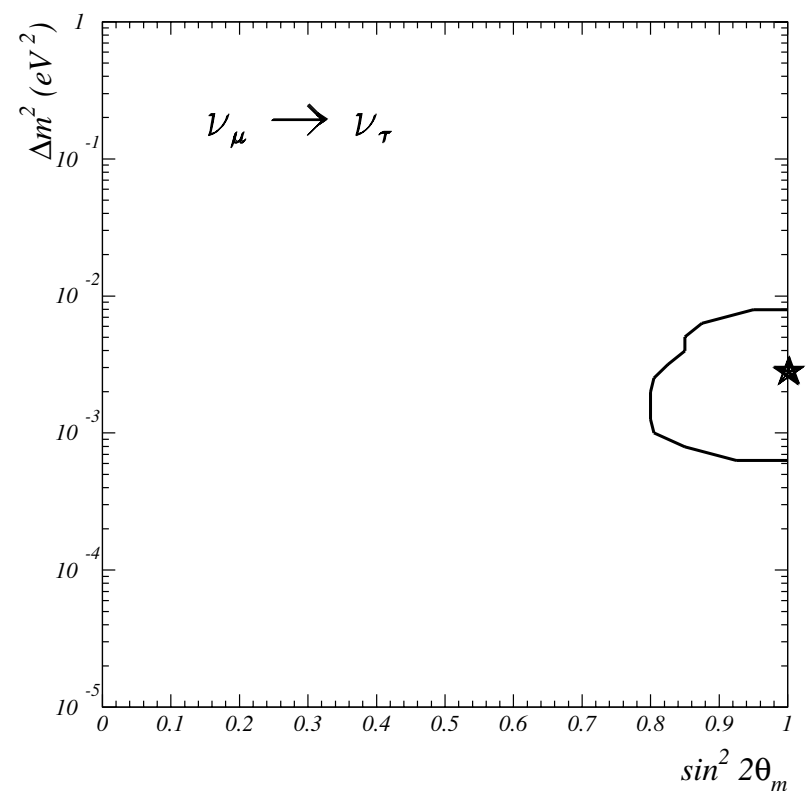

Fig. 12. The $90 \%$ c. l. region computed combining the angular distribution of UpThrough events, the low energy topologies and the high and low energy subsamples of UpThrough events. The star indicates the highest probability point in the physical region

(angular distribution only) is 27.6 for no oscillations and 9.5 at the point corresponding to the maximum probability.

In [19] we used the absolute rate of the UpThrough events to give further evidence in favor of the oscillation hypothesis. Assuming the Bartol flux, the rate data in this paper support oscillations and significantly reduce the allowed region. However, because of the uncertainty in the neutrino flux described in Sect. 5.2, we have chosen not to use the normalization. The situation is similar for arguments using the low energy events [18]; the oscillation evidence is stronger assuming the Bartol flux also for these samples.

\section{$7.2 \nu_{\mu} \rightarrow \nu_{\text {sterile }}$ oscillations}

As shown [19] the ratio Vertical/Horizontal can also be used to test the $\nu_{\mu} \rightarrow \nu_{\text {sterile }}$ oscillation hypothesis. For this channel the angular distribution of high energy events due to matter effects is quite similar to that for no oscillations. The measured value $R_{\text {meas }}=1.38$ should be compared to $R_{\tau}=1.61$ and $R_{\text {sterile }}=2.03$. These are the minimum values of the ratio for the oscillation hypotheses $\nu_{\mu} \rightarrow \nu_{\tau}$ and $\nu_{\mu} \rightarrow \nu_{\text {sterile }}$; in both cases, the optimal values of the parameters are $\sim 0.0023 \mathrm{eV}^{2}$ and maximal mixing.

For the $\nu_{\mu} \rightarrow \nu_{\tau}$ hypothesis, the one-sided probability of obtaining a value lower than $R_{\text {meas }}$ is $7.2 \%$. For $\nu_{\mu} \rightarrow \nu_{\text {sterile }}$, the corresponding probability is $1.5 \times 10^{-4}$, or 480 times smaller. The $\nu_{\mu} \rightarrow \nu_{\text {sterile }}$ hypothesis (with any mixing) is excluded at a confidenc level of about $99.8 \%$ with respect to the $\nu_{\mu} \rightarrow \nu_{\tau}$ hypothesis, with maximal mixing.

\section{Conclusions}

The MACRO experiment investigated atmospheric neutrinos in the oscillation parameter region anticipated in the MACRO proposal. Different analyses have been performed in different energy ranges and all the results are compatible with $\nu_{\mu} \rightarrow \nu_{\tau}$ oscillations. The preferred point in the oscillation parameter plane is $\sin ^{2} 2 \theta_{m}=1$ and $\Delta m^{2}=0.0023 \mathrm{eV}^{2}$, in agreement with our previous results and with the SuperKamiokande and Soudan 2 experiments. Our data are about $5 \sigma$ away from the no-oscillation hypothesis.

The most effective analyses concern three independent ratios which are essentially free of uncertainties due to the neutrino flux normalization. The three ratios are strongly coherent and point toward the oscillation. MACRO experimental data also provide strong constraints on the normalization of the atmospheric neutrino flux suggesting an increase in the high energy range of about $25 \%$ above the most recent calculations with the ICRC2001 fit [37]. Also in the low energy range our data suggest that the normalization should be increased of about $12 \%$ (see Table 3 ).

Acknowledgements. We gratefully acknowledge the support of the director and of the staff of the Laboratori Nazionali del Gran Sasso and the invaluable assistance of the technical staff of the Institutions participating in the experiment. We thank the Istituto Nazionale di Fisica Nucleare (INFN), the U.S. Department of Energy and the U.S. National Science Foundation for their generous support of the MACRO experiment. We thank INFN, ICTP (Trieste), WorldLab and NATO for providing fellowships and grants (FAI) for non Italian citizens.

\section{A Effective live-time estimate}

The acceptance of the detector varied during data taking because some elements were off during some periods and the efficiencies were not exactly constant. These time variations in the acceptance have been taken into account by estimating an effective live-time as described here.

In Fig. 13, the distribution of the rate of muon events is shown for the entire attico data-taking. The detector efficiency fluctuations do not prevent us from distinguishing three rate levels associated with the number of working, data-taking acquisition computers (1, 2 or 3 computers with 2,4 or 6 supermodules, respectively). This figure shows that the detector mainly operated in the full configuration (92.8\% of the time) .

In order to account for the effect of different run configurations on the effective live-time of the detector, we selected sets of "reference runs". These runs were characterized by having: the full configuration of the apparatus active; a duration greater than 4 hours with a dead time lower than $3 \%$; an average wire and strip efficiency on each module greater than $87 \%$ and $77 \%$, respectively; no scintillation counter turned off. A set of consecutive runs satisfying these criteria was selected with a periodicity of 


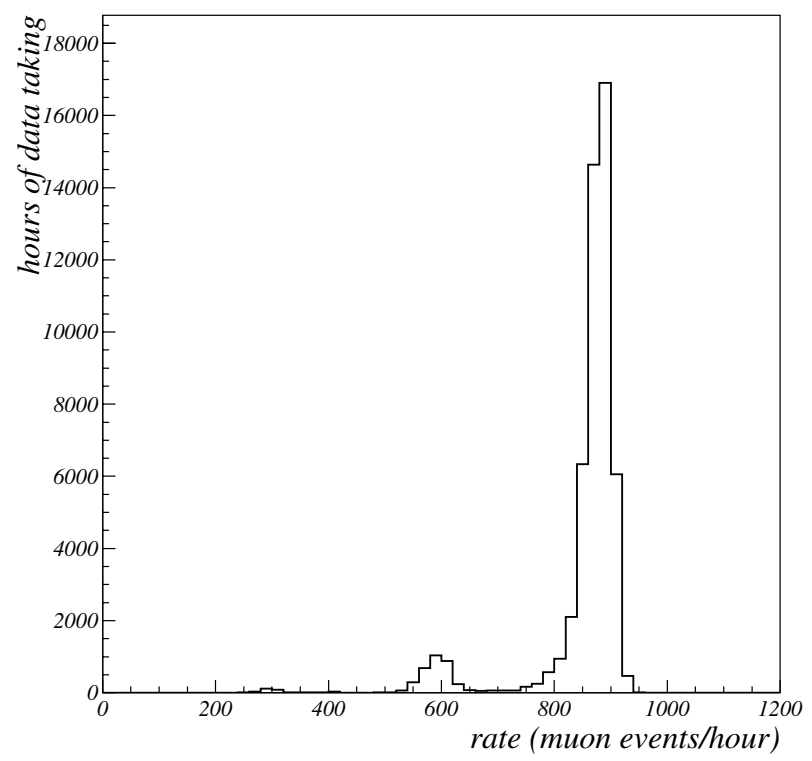

Fig. 13. Muon event rate distribution. Three rate levels (around 850,570 and 280 muon events/hour) are visible, due to different configurations of the detector $(6,4$ or 2 supermodules)

6 months (around January and July to account for the seasonal variation in the atmospheric muon rate [47]).

The effective live-time for a full data-taking sample, $L T_{\text {sample }}$, is estimated as

$$
L T_{\text {sample }}=\frac{N_{\text {sample }}}{N_{\text {ref }}} L T_{\text {ref }}
$$

where $N_{\text {ref }}$ is the number of tracks in the reference runs surviving the analysis cuts (except the final $1 / \beta$ requirement defining upward events), $N_{\text {sample }}$ is the number of tracks collected during the entire data-taking period and $L T_{\text {ref }}$ is the duration of all reference runs. This method is faster and simpler than estimating the efficiency of each detector component during the entire data-taking. We have also checked that the ratio in (3) does not depend on the zenith angle; therefore this method cannot affect the angular distribution measurement.

\section{B Two analyses for UpThrough events}

\section{Analysis A}

In this analysis an offline calibration of the scintillator system was used in order to reject badly performing scintillators and to cross-check the hardware calibrations described in [26]. Moreover, this offline calibration, described below, guaranteed a continuous check during the entire datataking period while standard calibrations were performed only weekly. Corrections to the parameters of the standard calibration (particularly the time offsets) were calculated by analyzing samples of about $2 \times 10^{5}$ single muons which provided adequate statistics on each TDC channel.

The 12-bit TDC counts were accepted in the range [50, 4050]. The time offsets for the single tanks were recalculated by means of an iterative procedure requiring $T o F$ compatible with relativistic tracks. The distribution of the difference between the time measured and the time expected from what was observed in other counters on the track was used to correct the offset of each TDC channel. The scintillation counters were rejected when the standard deviation of this distribution was greater than $10 \mathrm{~ns}$. The iterative procedure stopped when new corrections were smaller than $1 \mathrm{~ns}$. The distribution of the differences between the position calculated from the signal times observed at the ends of a scintillator and the position from the ST track has an $R M S$ of $12 \mathrm{~cm}$ corresponding to a time resolution of about 600 ps (Fig. 14).

Further analysis cuts were applied to remove backgrounds. The $\beta$ value was calculated when a track reconstructed in space intercepted at least two scintillators in different layers. If the released energy in a counter was smaller than $5 \mathrm{MeV}$ the adjacent hit counter was also considered. If a track intersected a cluster of adjacent counters, the signal time from the counter with the largest energy release was used. At this step of the analysis a minimum track length of $1 \mathrm{~m}$ was required in order to be safely above the scintillator time resolution.

The main discriminant of this analysis was the "position cut": the position along the counter measured by the ST system was required to agree within $\pm 70 \mathrm{~cm}( \pm 140 \mathrm{~cm}$ for tracks with $|\cos \theta|<0.2$ ) to that calculated from the signal times at the scintillator's ends.

Afterwards the $\beta$ estimate was done in two ways: for tracks intercepting only 2 counters, (2) was applied; for tracks crossing at least 3 counters the timing information was redundant and therefore $\beta$ was estimated from a linear fit of time versus scintillator position. In this case a $\chi^{2}$ cut

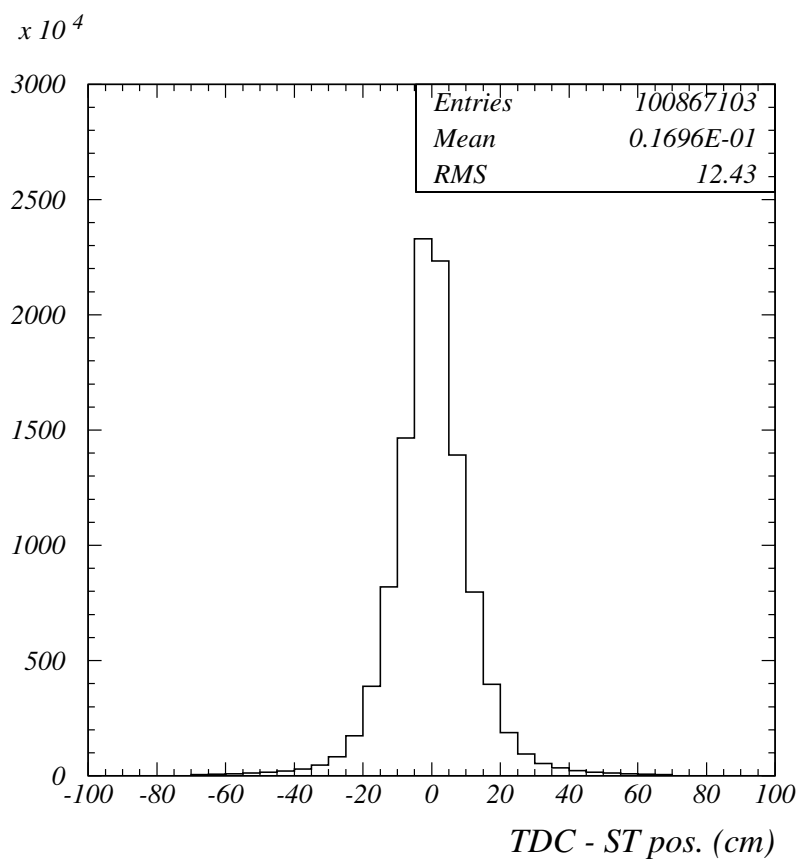

Fig. 14. Distribution of the difference of the position along the counter as calculated from the observed signal times at the ends of the scintillator and that given by the ST tracking 

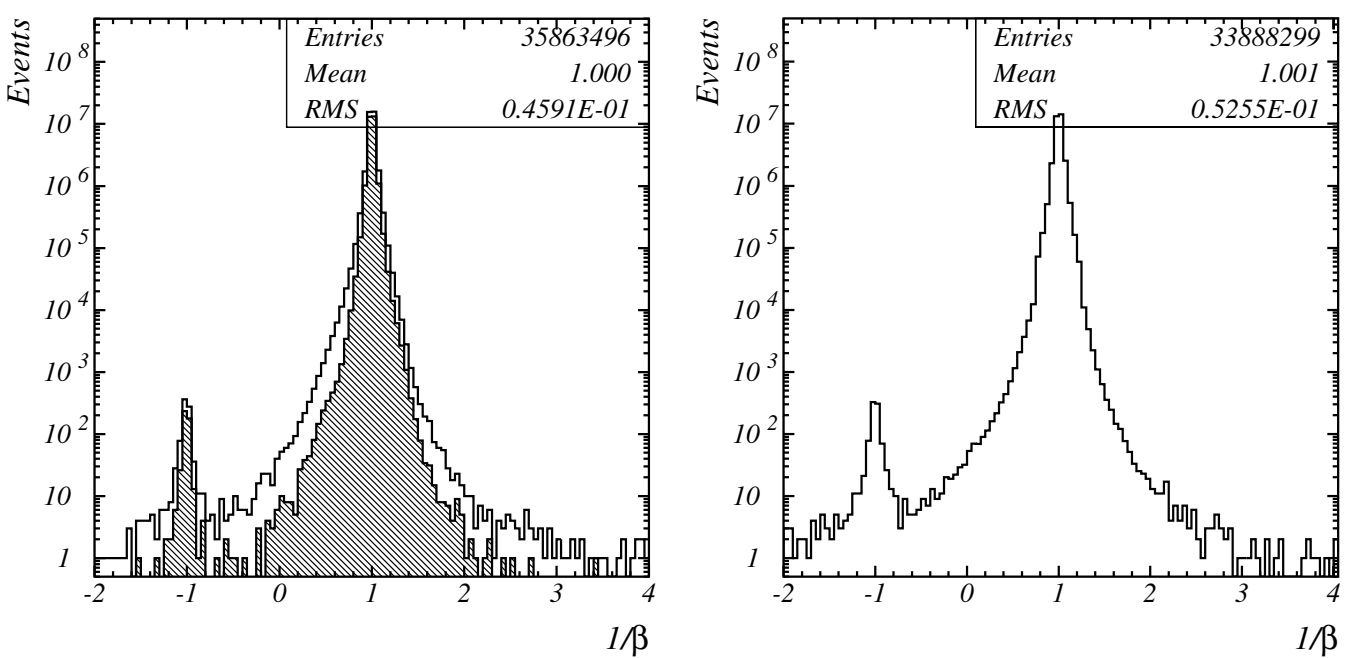

Fig. 15. $1 / \beta$ distribution for long tracks (at least two meters in the lower MACRO) for analyses A and B. The shaded region in the first distribution shows the "golden" events

was applied to discard poorly reconstructed tracks. The events with redundant time measurement $(\sim 50 \%$ of the total sample) had a particularly small background and they are considered a "golden" sample.

The analysis cuts were applied to the overall sample of events :

- the path-length between extreme counters was required to be larger than $2.5 \mathrm{~m}$;

- Teramo cut; horizontal events $(|\cos \theta|<0.1)$ were rejected if $170^{\circ}<\phi<250^{\circ}$, where $\phi$ is the azimuth angle.

The following cuts were applied to events where the $\beta$ value was estimated using only two scintillator counters:

- the track multiplicity was required to be less than 3 ;

- the difference between the ToF values obtained from the two TDC thresholds was required to be lower than $5 \mathrm{~ns}$;

- no more than one scintillator cluster not associated with the ST track was allowed;

- all tracks in an event were required to have the same sign of $\beta$.

A cut requiring a minimum amount of absorber to be crossed by the track ( $A B S$ cut) was applied in order to reduce the upward-going pion background [44]. In the first plot of Fig. 15 the $1 / \beta$ distribution is shown for the data from the full detector; it contains about 36 million atmospheric muons fulfilling all analysis requirements. There are 821 events in the signal range $[-1.25,-0.75]$. This is to be compared to 1062 events expected if there were no oscillations. The numbers of measured, expected, and background events are summarized in Table 1 . The systematics of this analysis have been discussed in $[14,15]$. The background due to incorrect $\beta$ measurements is estimated from the $1 / \beta$ distribution by considering two ranges close to the peak of upward-going muons $([-2,-1.2]$ and $[-0.7,-0.5])$. The events in these ranges are counted and the background events in the UpThrough signal range are estimated assuming a flat distribution of the background. Other backgrounds are estimated using simulations.

\section{Analysis B}

In this analysis the standard ERP time information was used without any recalibration. In each event, tracks joining all possible pairs of triggered scintillator clusters were initially considered. Then as described below several requirements were imposed until at most a single pair was selected. They were designed to ensure geometrical compatibility with a ST track and to give high quality timing information. If two or more scintillator pairs fulfilled these requirements and their distances of nearest approach to the ST track were not the same, the one with the nearer approach was chosen; otherwise, the pair with the longer track was selected.

1. Geometrical consistency of a ST track with the scintillator track was required. The distance between the two tracks had to be less than $50 \mathrm{~cm}$ in the $x-z$ view and $30 \mathrm{~cm}$ in the $y-z$ view. Furthermore the two tracks could not differ by more than $200 \mathrm{~cm}$ at the same height in either view.

2. To ensure that the $T o F$ was significantly larger than the scintillator time resolution, the distance between the scintillator clusters had to be greater than $250 \mathrm{~cm}$.

3. There could be no more than 5 scintillation counters not associated with the ST track.

4. The timing difference between the high and low scintillator pulse height thresholds had to be less than $2 \mathrm{~ns}$.

5. When many pairs of scintillator clusters were present in the same event, there was a hierarchy in the choice of the track based on the $1 / \beta$ value: tracks with $1 / \beta$ in the range $[0.75,1.25]$ were ranked highest; next, those in the range $[-1.25,-0.75]$; and finally tracks with other $1 / \beta$ values.

6. Only TDC counts in the range $[50,4050]$ were accepted.

7. Some micro-cuts were applied to remove a few runs or scintillation counters which generated many wrong $1 / \beta$ measurements. 
8. The $A B S$ cut required at least two meters of pathlength in lower MACRO. This cut removed also the In $U p$ events.

Finally the Teramo cut was applied: nearly horizontal tracks $(-0.1 \leq \cos \theta \leq 0)$ were rejected in the azimuth range $\left[180^{\circ}, 280^{\circ}\right]$. As a result of this last cut, 4 events were rejected in the $1 / \beta$ signal range. The second plot of Fig. 15 shows the $1 / \beta$ distribution after all cuts were applied. About 34 million events satisfied the analysis requirements and 870 events were in the signal range. The cuts affected essentially only the background and not the physical signal. Indeed the peak around $1 / \beta=+1$ (downward-going muons) is highly symmetric and only the $A B S$ cut reduced its size. Fitting the $1 / \beta$ distribution in the $[-6,-0.2]$ range to the sum of a straight line and a Gaussian permits us to estimate the residual background (43 events). The angular distribution of this background is estimated to be in agreement with that of the events outside the signal, in the ranges $[-6,-1.25]$ and $[-0.75,-0.2]$. Another 13 events are subtracted to take into account the presence of upward-going pions generated by atmospheric muons [44]. Finally the measured UpThrough signal is 814 events, to be compared to 1125 expected events (see Table 1) obtained by applying the analysis procedure to the no-oscillation simulated sample (see Sect. 5.2) and taking into account the effective livetime estimated by the reference run method.

\section{Analysis P with PHRASE system}

A third, completely independent analysis was made to check the efficiencies and the performances of the other analyses for the UpThrough sample [54]. A data subset collected in 2.2 years with the PHRASE system [25], designed for the detection of electron neutrinos from stellar collapses [55], was studied. The digitizing electronics and readout computers were completely different from those of the ERP system. Only the PMT signal fan-outs and the data logging computer were common between the two systems. The PHRASE electronics digitized the PMT signals with Flash Analog to Digital Converters (FADC) at $100 \mathrm{MHz}$. When the energy released in a counter exceeded $15 \mathrm{MeV}$ the FADC started to saturate on the signal from one or both ends of a scintillator. In this case, the sum of the signals from the two ends was no longer proportional to the signal charge and started to be logarithmically dependent on it. Energy deposits greater than $200 \mathrm{MeV}$ could be measured with a resolution of about 20\% [56]. The hit coordinate along the counter and its time were obtained from the rising edge of the PMT signals. At the beginning of each run, a start signal was sent to synchronize the internal clocks. The intrinsic precision in the measurement of the time difference between two counters is $1.6 \mathrm{~ns}$. A relevant difference from the analyses based on ERP data is that the activity of each electronic module serving one scintillator counter is independent of all the others. When data is read out of one module, no dead-time is introduced into any other one.

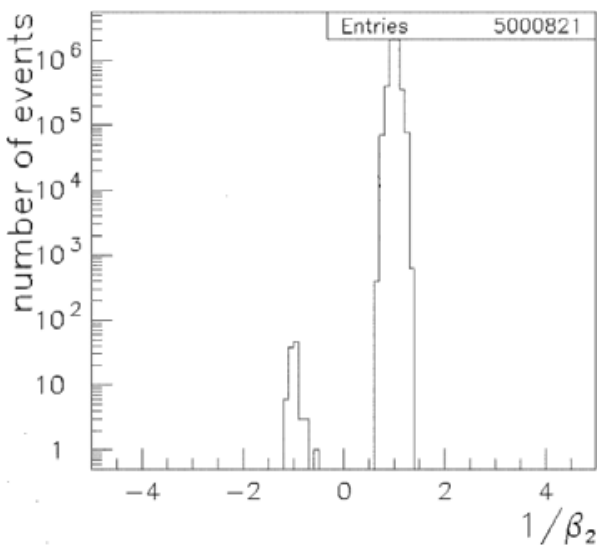

Fig. 16. Distribution of the $1 / \beta_{2}$ value (calculated using the extreme counters in the track) after the application of the cuts for analysis $\mathrm{P}$

A preliminary calibration was performed on a data subsample to estimate time offsets for each counter. These offsets (a few ns was typical) were calculated requiring $1 / \beta \sim 1$ for downward-going muons.

The identification of the muon flight direction was obtained using only the PHRASE information. To reject the multiple muon background only events with one scintillator cluster in each of three different layers were considered, and it was required that the distance of the intermediate cluster from the straight line connecting the extreme scintillator clusters was less than $1.5 \mathrm{~m}$. Then the best-fit straight line connecting the 3 clusters was reconstructed; this resulted in a sample of $7.7 \times 10^{6}$ tracks. To select a pure and completely independent sample of UpThrough events using only the PHRASE information, the following restrictive requirements were applied. The efficiency of this selection is expected to be poor.

1. The reconstructed energy in each scintillator was required to be greater than $7 \mathrm{MeV}$.

2. The distance between the best-fit straight line and each cluster was required to be less than $60 \mathrm{~cm}$.

3. The distance between scintillator clusters was required to be no less than $3 \mathrm{~m}$. This requirement implicitly contains the $A B S$ cut used by the other analyses to reduce the background of soft pions induced by downwardgoing muons; it reduced the detector acceptance by $15 \%$.

4. The time differences between each scintillator pair had to be consistent with a common flight direction.

5. In some runs, a few counters were not correctly synchronized resulting in times systematically earlier (or later) by multiples of $10 \mathrm{~ns}$. These counters were identified in each run and excluded from the analysis.

About $5.0 \times 10^{6}$ events survived, with two clear and well-separated peaks in the $1 / \beta$ distribution (see Fig. 16) giving 97 observed UpThrough events.

The MC described in Sects. 4 and 5.2, with the acceptance and the efficiencies of this analysis, yields an expected 147 Up Through events. The zenith angle distributions for data and simulation are shown in Fig. 17. 


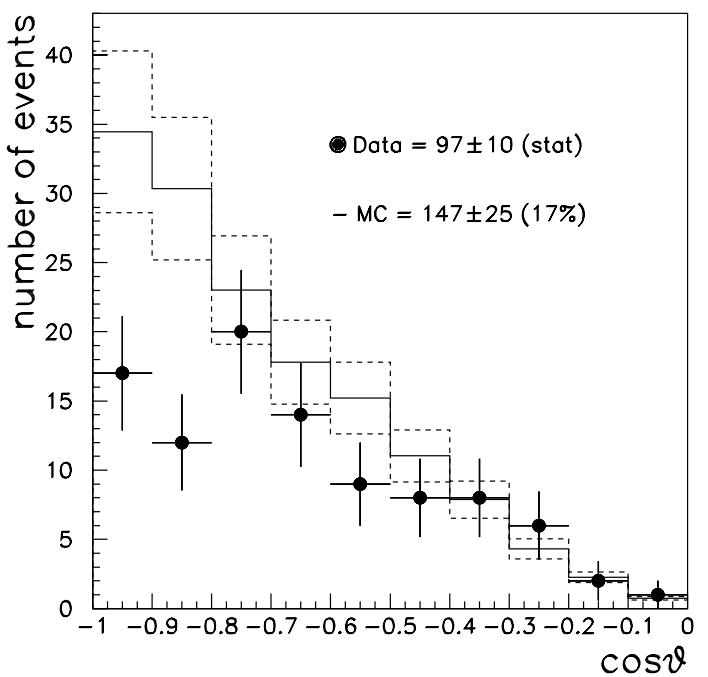

Fig. 17. Angular distribution of the 97 PHRASE UpThrough events (circles). The solid line corresponds to the MC prediction and the dashed line indicates the theoretical error

Comparing this sample with that selected by analysis A in the same periods of data-taking, we find that 82 events are common to both analyses, 4 events are only in analysis $\mathrm{P}$ and 61 events are only in analysis A. The relatively low efficiency of analysis $\mathrm{P}$ was expected; it is mainly an effect of cuts 2 and 3 . Since analysis $\mathrm{P}$ is a completely independent check of the ERP analyses, the $4 \mathrm{P}$-events missing in the A-sample represent a measurement of the inefficiency of the ERP analyses. Of these events :

- 2 events were lost because of a writing error on the standard data tapes.

- 1 event was lost because of a problem in the ST system.

- 1 event was lost because of a timing error in one counter of the ERP TDC system.

All these inefficiencies are accounted for in the efficiency evaluation of the ERP analyses. Thus analysis P identified no systematic effects which affect the number or the angular distribution of the Analyses A/B UpThrough sample.

\section{References}

1. B. Pontecorvo, J. Exp. Theor. Phys. 33, 549 (1957); J. Exp. Theor. Phys. 34, 247 (1958)

2. T.J. Haines et al., Phys. Rev. Lett. 57, 1986 (1986); D. Casper et al., Phys. Rev. Lett. 66, 2561 (1991); R. BeckerSzendy et al., Phys. Rev. D 46, 3720 (1992)

3. K.S. Hirata et al., Phys. Lett. B 205, 416 (1988); Phys. Lett. B 280, 146 (1992)

4. C. Berger et al., Phys. Lett. B 245, 305 (1990)

5. M. Aglietta et al., Europhys. Lett. 8, 611 (1989)

6. Y. Fukuda et al., Phys. Lett. B 335, 237 (1994)

7. Y. Fukuda et al., Phys. Rev. Lett. 81, 1562 (1998)

8. Y. Fukuda et al., Phys. Lett. B 467, 185 (1999)

9. Y. Fukuda et al., Phys. Rev. Lett. 82, 2644 (1999)

10. W.W. Allison et al., Phys. Lett. B 391, 491 (1997); Phys. Lett. B 449, 137 (1999); D.A. Petyt et al., Nucl. Phys. B (Proc. Suppl.) 110, 349 (2002); M. Sanchez et al., Phys. Rev. D 68, 11304 (2003)
11. C. De Marzo et al., Proposal for a large area detector dedicated to monopole search, astrophysics, and cosmic ray physics at the Gran Sasso Laboratory (1984)

12. F. Ronga (MACRO Collaboration), Proceedings of the Fifth International Symposium on Neutrino Telescopes, Venice, 285 (1993)

13. D.G. Michael (MACRO Collaboration), TAUP 93 Workshop, Nucl. Phys. B (Proc. Suppl.) 35, 235 (1994)

14. S. Ahlen et al., Phys. Lett. B 357, 481 (1995)

15. M. Ambrosio et al., Phys. Lett. B 434, 451 (1998)

16. P. Bernardini (MACRO Collaboration), Vulcano Workshop 1998, SIF Conference Proceedings 65, 539 (1999), also hepex/9809003

17. F. Ronga (MACRO Collaboration), Neutrino '98 Conference, Nucl. Phys. B (Proc. Suppl.) 77, 117 (1999), also hep-ex/9810008

18. M. Ambrosio et al., Phys. Lett. B 478, 5 (2000)

19. M. Ambrosio et al., Phys. Lett. B 517, 59 (2001)

20. M. Ambrosio et al., Nucl. Instrum. Methods A 492, 376 (2002)

21. M. Ambrosio et al., Phys. Lett. B 566, 35 (2003)

22. M. Ambrosio et al., Astrophys. J. 546, 1038 (2001)

23. M. Ambrosio et al., Astropart. Phys. 19, 1 (2003)

24. M. Ambrosio et al., Phys. Rev. D 60, 082002 (1999)

25. S. Ahlen et al., Nucl. Instrum. Methods A 324, 337 (1993)

26. M. Ambrosio et al., Nucl. Instrum. Methods A 486, 663 (2002)

27. M. Ambrosio et al., Phys. Rev. D 59, 012003 (1998); Astropart. Phys. 20, 145 (2003)

28. M. Ambrosio et al., Astropart. Phys. 8, 123 (1998)

29. T.K. Gaisser, M. Honda, Ann. Rev. Nucl. Part. Sci. 52, 153 (2002)

30. T. Montaruli, to appear in Plenary/Highlight/Rapporteur Proceedings of $28^{t h}$ International Cosmic Ray Conference, Tsukuba (2003), also hep-ph/0311289

31. V. Agrawal et al., Phys. Rev. D 53, 1314 (1996)

32. R. Engel et al., Proceedings of $28^{t h}$ International Cosmic Ray Conference, Tsukuba, 1603 (2003)

33. G. Battistoni et al., Astropart. Phys. 19, 269 (2003), Erratum 291

34. G. Battistoni et al., Proceedings of $28^{\text {th }}$ International Cosmic Ray Conference, Tsukuba, 1399 (2003)

35. M. Honda et al., Phys. Rev. D 64, 053011 (2001)

36. M. Honda et al., Proceedings of $28^{\text {th }}$ International Cosmic Ray Conference, Tsukuba, 1415 (2003)

37. T.K. Gaisser et al., Proceedings of $27^{\text {th }}$ International Cosmic Ray Conference, Hamburg, 1643 (2001)

38. J. Alcaraz et al., Phys. Lett. B 490, 27 (2000); Phys. Lett. B 494, 193 (2000)

39. T. Sanuki et al., Astrophys. J. 545, 1135 (2000)

40. K. Asakimori et al. (JACEE Collaboration), Astrophys. J. 502, 278 (1998)

41. A.V. Apanasenko et al. (RUNJOB Collaboration), Astropart. Phys. 16, 13 (2001); M. Furukawa et al. (RUNJOB Collaboration), Proceedings of $28^{\text {th }}$ International Cosmic Ray Conference, Tsukuba, 1837 (2003)

42. W. Lohmann et al., CERN-EP/85-03 (1985)

43. R. Brun et al., CERN Publication DD/EE/84-1 (1992)

44. M. Ambrosio et al., Astropart. Phys. 9, 105 (1998)

45. M. Glück et al., Z. Phys. C 67, 433 (1995)

46. P. Lipari, Nucl. Phys. B (Proc. Suppl.) 91, 159 (2001)

47. M. Ambrosio et al., Astropart. Phys. 7, 109 (1997)

48. M. Ambrosio et al., Eur. Phys. J. C 25, 511 (2002) 
49. P. Lipari et al., Phys. Rev. Lett. 74, 4384 (1995)

50. Private Communication from MINOS Collaboration

51. M. Apollonio et al., Eur. Phys. J. C 27, 331 (2003)

52. B. Roe, Probability and Statistics in Experimental Physics, (Springer-Verlag, Berlin 1992) 128
53. G.J. Feldman, R.D. Cousins, Phys. Rev. D 57, 3873 (1998)

54. M. Spurio (MACRO Collaboration), Nucl. Phys. B (Proc. Suppl.) 85, 37 (2000), also hep-ex/9908066

55. S. Ahlen et al., Astropart. Phys. 1, 11 (1992)

56. M. Ambrosio et al., Astropart. Phys. 6, 113 (1997) 